\title{
Visible Light-Promoted Formation of C-B and C-S Bonds under Metal- and Photocatalyst-Free Conditions
}

\author{
Lena Blank ${ }^{\mathrm{a}}$ \\ Maurizio Fagnonib \\ Stefano Protti ${ }^{b}$ \\ Magnus Rueping*a ${ }^{(1)}$ \\ a Institute of Organic Chemistry, RWTH Aachen University, Landoltweg 1, \\ 52074 Aachen, Germany \\ magnus.rueping@rwth-aachen.de \\ b PhotoGreen Lab, Department of Chemistry, University of \\ Pavia, Viale Taramelli, 12, 27100 Pavia, Italy \\ Published as part of the 50 Years SYNTHESIS - Golden Anniversary Issue
}

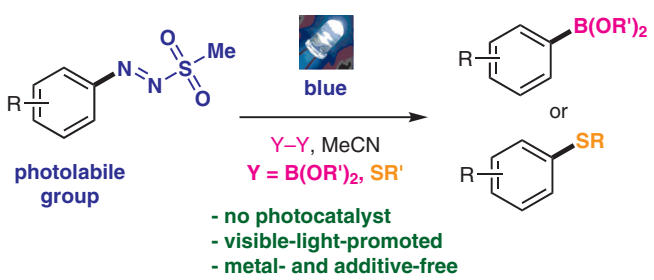

\author{
Received: 30.11 .2018 \\ Accepted: 10.12 .2018 \\ Published online: 06.02.2019 \\ DOI: 10.1055/s-0037- 1611648; Art ID: ss-2018-z0804-op \\ License terms: cc)
}

Abstract A green, efficient, photoinduced synthesis of arylboronic esters and aryl sulfides has been developed. Bench stable arylazo sulfones were used as radical precursors for a photocatalyst- and additive-free carbon-heteroatom bond formation under visible light. The protocols are applicable to a wide range of substrates, providing products in good yields.

Key words arylazo sulfones, aryl radicals, arylboronic esters, aryl sulfides

The development of mild and sustainable protocols for carbon-heteroatom bond formation has received an impressive attention in recent years. Indeed, aryl boronates and aryl sulfides play a key role in organic synthesis, ${ }^{1}$ catalysis, ${ }^{2}$ material science, ${ }^{3}$ and especially medicinal chemistry. ${ }^{4}$ Many natural products, drugs, and crop-protection substances bear sulfur-containing functional groups. ${ }^{5}$ In addition, since the introduction of Bortezomib in 2003 by the FDA (US Federal Drug Administration) ${ }^{6}$ for treating multiple myeloma, an increasing interest in boronic acids as drug candidates has developed. ${ }^{7}$

Classical methods for the generation of carbon-heteroatom bonds involve thermal reactions with organometal derivatives, ${ }^{8}$ the use of transition-metal catalysts in combination with expensive often air-sensitive ligands, strong bases, and additives in excess amounts. ${ }^{9}$ In order to reduce costs and contaminations with heavy metals, the development of more efficient and environmentally friendly alternative routes is highly desirable.

Several metal-free photoinduced borylation protocols of haloarenes, ${ }^{10}$ quaternary arylammonium salts, aryl triflates, ${ }^{11}$ and carboxylic acids ${ }^{12}$ as substrates have been pub- lished recently (Scheme 1, a-c). Such methods feature the advantage of avoiding the use of late transition metals. Nevertheless, they still suffer from several drawbacks, like the need of ultraviolet light irradiation and additives such as NaI, $N, N, N^{\prime}, N^{\prime}$-tetramethyldiaminomethane (TMDAM), or $\mathrm{Cs}_{2} \mathrm{CO}_{3}$ in stochiometric amounts. An alternative for the preparation of arylboronic esters and aryl sulfides under photochemical conditions, takes advantage of aryl halides and aryldiazonium salts (Scheme $1, \mathrm{~d}-\mathrm{f}),{ }^{13}$ which are widely used as aryl radical sources in photochemistry. The activation of aryldiazonium salts under visible light requires a photocatalyst (PC), since the aryl radical can only be formed by single-electron transfer (SET) from the excited state of the photocatalyst to the diazonium salt.
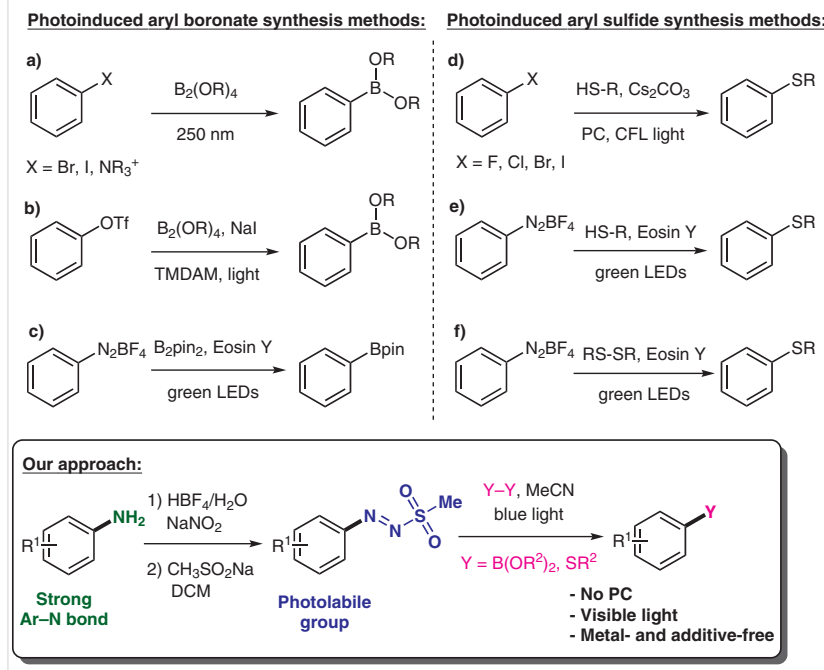

Scheme 1 Representative methods for the synthesis of arylboronic esters and aryl sulfides 
Recently published protocols revealed that arylazo sulfones can be successfully used in the metal-free arylation of heterocycles and unactivated arenes without the need of additives. ${ }^{14}$ Herein, we report the application of arylazo sulfones in the photocatalyst-, metal- and additive-free, visible-light-driven synthesis of aryl sulfides and aryl boronates. ${ }^{15}$

The present protocol is a sustainable approach that exploit bench stable arylazo sulfones, easily prepared from anilines, and allow the wavelength selective generation of aryl radicals and aryl cations. ${ }^{14,15}$

At the beginning of our studies, several reaction conditions were evaluated in order to determine the ideal setup for the photocatalyst-free borylation of arylazo sulfones (Table 1). Initially, a solution of arylazo sulfone $\mathbf{1 f}$, bis(pinacolato)diboron $\left[\mathrm{B}(\mathrm{pin})_{2}, \mathbf{2 a}\right]$, with $\mathrm{NaI}$ and TMDAM as additives in acetonitrile was stirred at room temperature. No product formation was observed neither in the absence of light (Table 1 , entry 1 ) nor when exposing the solution to daylight (entry 2). Only traces of the aryl-B(pin) 3f were obtained using 12 W LEDs for 12 hours (entry 3 ). However, $\mathbf{3 f}$ was isolated in $59 \%$ yield using high power $24 \mathrm{~W}$ blue LEDs (entry 4). Also, the H150 blue Kessil lamp ( $34 \mathrm{~W}$ ) was tested for irradiation, increasing the yield up to $79 \%$ (entry 6). Reactions without NaI and TMDAM showed that there is no need for additives to form the aryl boronate $3 \mathbf{f}$ in $56 \%$ yield, when using high power blue LEDs (entry 5). The yield was further improved to $78 \%$ by using a blue $34 \mathrm{~W}$ Kessil lamp (entry 7). Other polar solvents like methanol and an aceto-

Table 1 Reaction Optimization for the Photocatalyst-Free Aryl Borylation $^{\mathrm{a}}$

\begin{tabular}{|c|c|c|c|}
\hline Entry & Additive & Light source & Yield (\%) \\
\hline 1 & $\mathrm{Nal} / \mathrm{TMDAM}$ & in the dark & - \\
\hline 2 & Nal/TMDAM & daylight & - \\
\hline 3 & Nal/TMDAM & blue LEDs $12 \mathrm{~W}$ & traces \\
\hline 4 & Nal/TMDAM & blue LEDs $24 \mathrm{~W}$ & 59 \\
\hline 5 & - & blue LEDs $24 \mathrm{~W}$ & 56 \\
\hline 6 & Nal/TMDAM & Kessil lamp 34 W & 79 \\
\hline 7 & - & Kessil lamp 34 w & 78 \\
\hline $8^{b}$ & - & Kessil lamp 34 W & 59 \\
\hline $9^{c, d}$ & - & Kessil lamp 34 W & 61 \\
\hline
\end{tabular}

a Reaction conditions: $1 f(0.1 \mathrm{mmol}), 2 \mathrm{a}(0.2 \mathrm{mmol})$, Nal (50 mol\%), TMDAM (1 equiv), MeCN, r.t., 12 h, yield after purification by column chromatography.

b Reaction was carried out in $\mathrm{MeOH}$.

c Reaction was carried out in $\mathrm{MeCN} / \mathrm{H}_{2} \mathrm{O}(9: 1)$.

d Yield, determined via ${ }^{1} \mathrm{H}$ NMR spectroscopy using dimethyl sulfone as internal standard. nitrile/water mixture (9:1) gave no improvement on the yield (entries 8 and 9). The best results were obtained in acetonitrile using $34 \mathrm{~W}$ blue light lamp for irradiation (entry 7).

Based on the optimised conditions, the substrate scope was examined using different arylazo sulfones and borylating agents (Scheme 2). We were pleased to see that the photoinduced aryl borylation tolerates the presence of halides (3b-e), electron-donating (3f-g), and electron-withdrawing substituents (3h-o) on the aromatic moiety of the arylazo sulfones. In all cases, moderate to good yields were achieved (up to 78\%). In addition, substrates containing potentially light-reactive substituents such as aryl ketone (3h) and biaryl (3i) were also appropriate radical precursors to give the resulting product under the described reaction conditions. Gratifyingly, arylazo sulfones bearing a bromide and iodide as substituent underwent also borylation (3d and 3e), without cleavage of the carbon-halogen bond.

Next, different borylating agents were studied. The results showed that compared to $B_{2} \operatorname{pin}_{2}$ (2a), bis(neopentyl glycolato)diboron (2b), and bis(hexylene glycolato)diboron (2c) gave slightly lower yields for the respective products 3p (64\%) and 3q (56\%). The borylation of 4-chlorophenylazo sulfone using bis(catecholato)diboron (2d) was also feasible.

We thus extended our investigation to the synthesis of thioethers, the visible light-mediated thiolation of arylazo sulfones was approached in the presence of disulfides as the thiolating reagent (Scheme 3). The scope was evaluated by employing various arylazo sulfones bearing halides, cyano, nitro, pentafluorosulfide, and keto groups $(\mathbf{5 a}-\mathbf{j})$. The products were obtained in moderate to good yields. Additionally, different ortho- and meta-substituents were tolerated $(\mathbf{5 k}-\mathbf{m})$. Diethyl sulfide (4b) and diphenyl sulfide (4c) were also successful in the photocatalyst-free thioether synthesis leading to $\mathbf{5 n}$ and $\mathbf{5 o}$ in $50 \%$ and $46 \%$ yield, respectively.

The proposed mechanism for the synthesis of aryl sulfides and boronates is depicted in Scheme 4. Irradiation of $\mathbf{1}$ with blue light $(\lambda=420 \mathrm{~nm})$ leads to the excited ${ }^{1} \mathrm{n} \pi^{*}$-state $\mathbf{1}^{*}$ (path a). Subsequent homolytic cleavage of the S-N bond (path b) affords the aryl radical 6 and methanesulfonyl radical 7. ${ }^{14}$ Such radicals are trapped by the diboron/disulfide $(\mathrm{Y}-\mathrm{Y})$ reagent $\mathbf{2}$ to give products $\mathbf{3}$ or $\mathbf{5}$ and the boron/sulfide radical ( $\left.\mathrm{Y}^{*}\right) \mathbf{8}$ (path $\mathrm{c}$ and $\mathrm{f}$ ). The combination between aryl and X-radicals to form $\mathbf{3}$ or $\mathbf{5}$ is also possible (path d). Hydrogen atom abstraction by the generated radicals from the solvent to give arenes 9 and sulfinic acid (10) could also take place as a secondary path (path g). Finally, the recombination of the aryl $\mathbf{6}$ and methanesulfonyl radical $\mathbf{7}$ leads to a second possible by-product sulfone 12 (path e, see SI).

In summary, we have developed an efficient, green, photoinduced aryl borylation and aryl thioether formation protocol with arylazo sulfones as aryl radical precursors. 


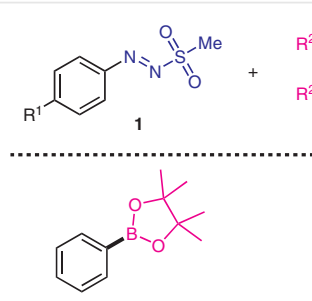

3a, $53 \%$

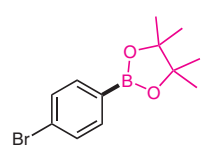

3d, $60 \%$<smiles>COc1ccc(B2OC(C)(C)C(C)(C)O2)cc1</smiles>

3g, $67 \%$<smiles>CC1(C)OB(c2ccc([N+](=O)[O-])cc2)OC1(C)C</smiles>

3j, $54 \%$<smiles>CCOC(=O)c1ccc(B2OC(C)(C)C(C)(C)O2)cc1</smiles>

$3 \mathrm{~m}, 55 \%$<smiles>Cc1ccc(B2OCC(C)(C)CO2)cc1</smiles>

3p, $64 \%$

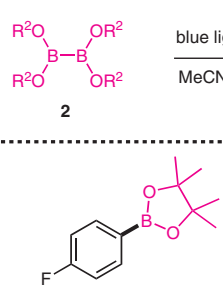

3b, $54 \%$<smiles>CC1(C)OB(c2ccc(I)cc2)OC1(C)C</smiles>

3e, $51 \%$<smiles>CC(=O)c1ccc(B2OC(C)(C)C(C)(C)O2)cc1</smiles>

3h, $52 \%$<smiles>CC1(C)OB(c2ccc(S(F)(F)F)cc2)OC1(C)C</smiles>

3k, $63 \%$<smiles>COC(=O)c1ccc(B2OC(C)(C)C(C)(C)O2)cc1</smiles>

3n, $57 \%$<smiles>Cc1ccc(B2OCC(C)(C)C(C)O2)cc1</smiles>

$3 q, 56 \%$

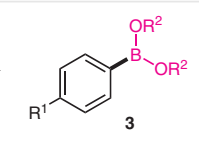

$\overbrace{-1}^{B+1}$

3c, $69 \%$<smiles>Cc1ccc(B2OC(C)(C)C(C)(C)O2)cc1</smiles><smiles>CC1(C)OB(c2ccc(-c3ccccc3)cc2)OC1(C)C</smiles><smiles>CC1(C)OB(c2ccc(C#N)cc2)OC1(C)C</smiles>

3I, $45 \%$<smiles>CC1(C)OB(c2cccc3ccccc23)OC1(C)C</smiles>

3o, $52 \%$

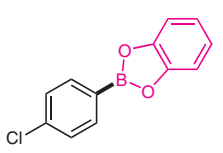

3r, $37 \%^{a}$

Scheme 2 Substrate scope of the photoinduced aryl borylation. Reagents and conditions: $\mathbf{1}(0.1 \mathrm{mmol}), \mathbf{2}(0.2 \mathrm{mmol}), \mathrm{MeCN}$, r.t., $\mathrm{H} 150$ Kessil lamp (blue light $34 \mathrm{~W}$ ), 12 h; yields after purification by column chromatography. ${ }^{\text {a }}$ Yield determined via ${ }^{1} \mathrm{H}$ NMR using dimethyl sulfone as internal standard, confirmed by MS (EI): $m / z=230$ (87).

These stable and easy to handle aryldiazonium salt derivatives show a high photoreactivity under blue light irradiation. The scope of the borylation reaction includes both, different aryl derivatives and different diboron esters, providing products in moderate to good yields. In addition, this simple approach could also be transferred to the aryl sulfide synthesis with disulfides.

Unless otherwise noted, all reagents were obtained from commercial suppliers. Organic solvents were purified from by solvent purification system MBraun SPS-5 and stored over molecular sieves under argon. Diazonium salts were synthesised by following a known procedure ${ }^{16}$ and purified by recrystallisation from acetone/Et $\mathrm{t}_{2} \mathrm{O}$. For irradiation of the reaction mixtures, different light sources were used: a) blue LED

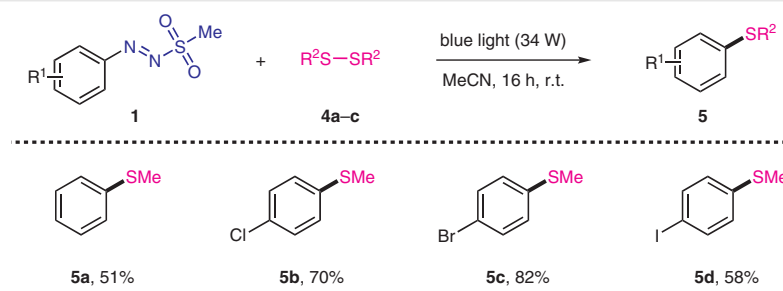<smiles>Cc1ccc(C)cc1</smiles><smiles>COc1ccc(OC)cc1</smiles><smiles>N#Cc1ccc(S)cc1</smiles><smiles>O=[N+]([O-])c1ccc([AsH3])cc1</smiles><smiles>[Mg]c1ccc(S)cc1</smiles><smiles>Cc1ccc(C(N)=O)cc1</smiles>

$5 \mathrm{i}, 58 \%$ 5j, $74 \%$<smiles>Cc1cccc([N+](=O)[O-])c1</smiles><smiles>CCc1ccccc1</smiles>

$5 \mathrm{~m}, 54 \%$
$5 \mathrm{~g}, 66 \%$

5 h, $63 \%$<smiles>COc1ccccc1C(C)=O</smiles><smiles>Cc1ccccc1C#N</smiles>

5 k, $50 \%$

$51,72 \%$

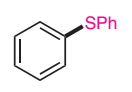

$50,46 \%$

Scheme 3 Substrate scope of the photoinduced synthesis of aryl sulfides. Reagents and conditions: $1(0.2 \mathrm{mmol}), \mathbf{4}(0.4 \mathrm{mmol}), \mathrm{MeCN}$, r.t., $\mathrm{H} 150$ Kessil lamp (blue light $34 \mathrm{~W}$ ), $12 \mathrm{~h}$. Yields after purification by column chromatography.
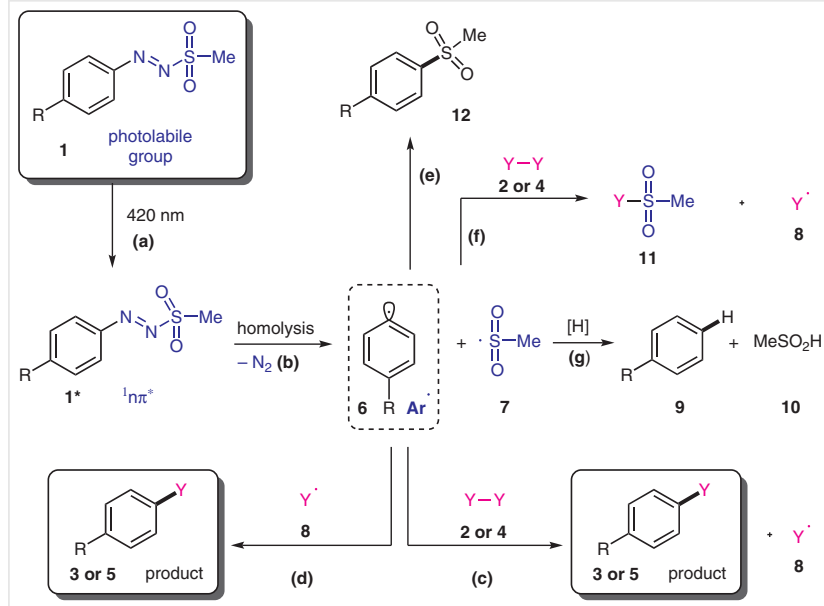

Scheme 4 Proposed mechanism for the PC-free photoinduced borylation and aryl sulfide formation via arylazo sulfones as stable radical precursors

stripes (12W, $24 \mathrm{~W}$ ) and b) H150 Kessil lamp (34 W, $\lambda=420 \mathrm{~nm}$ ). The reaction was cooled using a small ventilator, placed at $3 \mathrm{~cm}$ from the reaction vessels. A shield covered with aluminum foil was placed in front of the reaction setup to protect eyes from light irradiation. Solvents for chromatography were technical grade and distilled prior to use. TLC was carried out on Merck aluminum support plates Silica gel $60 \mathrm{~F}_{254}$. Visualisation was achieved under a UV mineral light or stained using cer-molybdato phosphoric acid (CAM). Column chromatography was performed using silica gel Merck $60(0.2-0.063 \mathrm{~mm})$. ${ }^{1} \mathrm{H}$ NMR spectra were recorded on Varian $400 \mathrm{MHz}$ and $600 \mathrm{MHz}$ 
spectrometers at 400 and $600 \mathrm{MHz} .{ }^{13} \mathrm{C}$ NMR spectra were similarly recorded at 100 or $150 \mathrm{MHz}$, using a broadband decoupled mode. ${ }^{11} \mathrm{~B}$ NMR spectra were measured at $128 \mathrm{MHz}$ and ${ }^{19} \mathrm{~F}$ NMR measurements were performed at $376 \mathrm{MHz}$ on Varian $400 \mathrm{MHz}$ and Mercury 300 $\mathrm{MHz}$ spectrometers, respectively. NMR chemical shifts ( $\delta$ ) are reported in parts per million (ppm) relative to residual proton signals in CD$\mathrm{Cl}_{3}(\delta=7.26,77.16 \mathrm{ppm})$. Coupling constants $(J)$ are reported in hertz $(\mathrm{Hz})$ and refer to apparent multiplicities. Standard abbreviations are used for the multiplicities. Mass spectra (EI-MS, $70 \mathrm{eV}$ ) were conducted on a Finnigan SSQ 7000 spectrometer. HRMS were recorded on a Thermo Scientific TQ Orbitrap XI spectrometer. IR spectra were recorded on an Jasco FT/IR-420 spectrophotometer and are reported in terms of frequency of absorption $\left(\mathrm{cm}^{-1}\right)$.

\section{Synthesis of Arylazosulfones 1; ${ }^{14 a}$ General Procedure}

To a cooled $\left(0^{\circ} \mathrm{C}\right)$ suspension of the appropriative diazonium salt ( 1.00 equiv, $0.30 \mathrm{M}$ ) in anhyd $\mathrm{CH}_{2} \mathrm{Cl}_{2}$ was added $\mathrm{MeSO}_{2} \mathrm{Na}$ (1.00 equiv) in one portion. The temperature was allowed to rise to r.t. overnight. The resulting mixture was filtered, and the obtained solution evaporated. The crude solid was purified by either dissolving in cold $\mathrm{CH}_{2} \mathrm{Cl}_{2}$ and precipitation by adding $n$-hexane or column chromatography.

\section{1-(Methylsulfonyl)-2-(phenyl)diazene (1a)}

Reaction of benzenediazonium tetrafluoroborate $(300 \mathrm{mg}$, $1.56 \mathrm{mmol}$ ) and $\mathrm{MeSO}_{2} \mathrm{Na}$ (160 mg, $1.56 \mathrm{mmol}, 1.00$ equiv) in $\mathrm{CH}_{2} \mathrm{Cl}_{2}$ $\left(5.00 \mathrm{~mL}, 0.3 \mathrm{M}\right.$ ) gave $1 \mathrm{a}\left(185 \mathrm{mg}, 9.93 \cdot 10^{-1} \mathrm{mmol}, 65 \%\right)$ after recrystallisation from cold $\mathrm{CH}_{2} \mathrm{Cl}_{2} / n$-hexane as a yellow solid.

${ }^{1} \mathrm{H} \mathrm{NMR}\left(400 \mathrm{MHz}, \mathrm{CDCl}_{3}\right): \delta=7.95(\mathrm{dd}, J=8.4,1.1 \mathrm{~Hz}, 2 \mathrm{H}), 7.70-7.65$ $(\mathrm{m}, 1 \mathrm{H}), 7.58(\mathrm{t}, J=7.6 \mathrm{~Hz}, 2 \mathrm{H}), 3.23(\mathrm{~d}, J=3.3 \mathrm{~Hz}, 3 \mathrm{H})$.

The NMR data were in accordance with the literature. ${ }^{14 a}$

\section{1-(Methylsulfonyl)-2-(4-fluorophenyl)diazene (1b)}

Reaction of 4-fluorobenzenediazonium tetrafluoroborate $(300 \mathrm{mg}$, $1.43 \mathrm{mmol}$ ) and $\mathrm{MeSO}_{2} \mathrm{Na}$ (146 mg, $1.43 \mathrm{mmol}, 1.00$ equiv) in $\mathrm{CH}_{2} \mathrm{Cl}_{2}$ (5.00 mL, $0.30 \mathrm{M}$ ) gave $\mathbf{1 b}$ (197 mg, 9.76.10-1 mmol, 68\%) after recrystallisation from cold $\mathrm{CH}_{2} \mathrm{Cl}_{2} / n$-hexane as a yellow solid; $\mathrm{mp} 66.7-$ $67.0^{\circ} \mathrm{C}$ (dec.).

IR (KBr, neat): 3025, 2942, 1587, 1492, 1332, 1227, 1137, 954, 892, $851 \mathrm{~cm}^{-1}$.

${ }^{1} \mathrm{H}$ NMR (400 MHz, $\mathrm{CDCl}_{3}$ ): $\delta=7.99$ (ddd, $J=8.2,5.1,2.6 \mathrm{~Hz}, 2 \mathrm{H}$ ), 7.31-7.19 (m, $2 \mathrm{H}), 3.20(\mathrm{~d}, J=7.2 \mathrm{~Hz}, 3 \mathrm{H})$.

${ }^{13} \mathrm{C} \mathrm{NMR}\left(101 \mathrm{MHz}, \mathrm{CDCl}_{3}\right): \delta=165.5,145.5,127.2,116.9,34.8$.

${ }^{19} \mathrm{~F}$ NMR $\left(376 \mathrm{MHz}, \mathrm{CDCl}_{3}\right): \delta=-101.0$.

MS (EI): $m / z(\%)=123$ (27), 95 (100).

\section{1-(Methylsulfonyl)-2-(4-Chlorophenyl)diazene (1c)}

Reaction of 4-chlorobenzenediazonium tetrafluoroborate $(1.20 \mathrm{~g}$, $5.30 \mathrm{mmol}$ ) and $\mathrm{MeSO}_{2} \mathrm{Na}$ (541 mg, $5.30 \mathrm{mmol}, 1.00$ equiv) in $\mathrm{CH}_{2} \mathrm{Cl}_{2}$ $(17.5 \mathrm{~mL}, 0.30 \mathrm{M})$ gave $1 \mathrm{c}(584 \mathrm{mg}, 2.67 \mathrm{mmol}, 51 \%)$ after recrystallisation from cold $\mathrm{CH}_{2} \mathrm{Cl}_{2} / n$-hexane as a yellow solid.

${ }^{1} \mathrm{H} \mathrm{NMR}\left(600 \mathrm{MHz}, \mathrm{CDCl}_{3}\right): \delta=7.91(\mathrm{~d}, J=8.6 \mathrm{~Hz}, 2 \mathrm{H}), 7.56(\mathrm{~d}, J=8.6$ $\mathrm{Hz}, 2 \mathrm{H}), 3.22$ (s, $3 \mathrm{H})$.

The NMR data were in accordance with the literature. ${ }^{14 a}$

\section{1-(Methylsulfonyl)-2-(4-Bromophenyl)diazene (1d)}

Reaction of 4-bromobenzenediazonium tetrafluoroborate $(1.00 \mathrm{~g}$, $3.69 \mathrm{mmol}$ ) and $\mathrm{MeSO}_{2} \mathrm{Na}$ (377 mg, $3.69 \mathrm{mmol}, 1.00$ equiv) in $\mathrm{CH}_{2} \mathrm{Cl}_{2}$ $(12.3 \mathrm{~mL}, 0.30 \mathrm{M})$ gave $\mathbf{1 d}(533 \mathrm{mg}, 2.03 \mathrm{mmol}, 55 \%)$ after recrystallisation from cold $\mathrm{CH}_{2} \mathrm{Cl}_{2} / n$-hexane as a yellow solid.

${ }^{1} \mathrm{H} \mathrm{NMR}\left(600 \mathrm{MHz}, \mathrm{CDCl}_{3}\right): \delta=7.83(\mathrm{~d}, J=8.7 \mathrm{~Hz}, 2 \mathrm{H}), 7.73(\mathrm{~d}, J=8.7$ $\mathrm{Hz}, 2 \mathrm{H}), 3.22$ (s, $3 \mathrm{H})$.

The NMR data were in accordance with the literature. ${ }^{14 \mathrm{~b}}$

\section{1-(Methylsulfonyl)-2-(4-Iodophenyl)diazene (1e)}

Reaction of 4-iodobenzenediazonium tetrafluoroborate (497 mg, $1.56 \mathrm{mmol}$ ) and $\mathrm{MeSO}_{2} \mathrm{Na}$ (160 mg, $1.56 \mathrm{mmol}, 1.00$ equiv) in $\mathrm{CH}_{2} \mathrm{Cl}_{2}$ (5.20 mL, $0.30 \mathrm{M})$ gave 1 e $\left(212 \mathrm{mg}, 6.85 \cdot 10^{-1} \mathrm{mmol}, 44 \%\right)$ after recrystallisation from cold $\mathrm{CH}_{2} \mathrm{Cl}_{2} / n$-hexane) as an orange solid; mp 132.0$132.4^{\circ} \mathrm{C}$ (dec.).

IR (KBr, neat): 3037, 2928, 2074, 1474, 1316, 1139, 952, 887, $830 \mathrm{~cm}^{-1}$. ${ }^{1} \mathrm{H}$ NMR (600·MHz, $\left.\mathrm{CDCl}_{3}\right): \delta=7.95(\mathrm{~d}, J=8.6 \mathrm{~Hz}, 2 \mathrm{H}), 7.65$ (d, $J=8.6$ $\mathrm{Hz}, 2 \mathrm{H}), 3.22$ (s, $3 \mathrm{H})$.

${ }^{13} \mathrm{C}$ NMR $\left(151 \mathrm{MHz}, \mathrm{CDCl}_{3}\right): \delta=148.2,139.2,125.6,103.6,34.8$.

MS (EI): $m / z(\%)=76$ (75), 203 (100), 231 (47).

\section{1-(Methylsulfonyl)-2-(4-Methylphenyl)diazene (1f)}

Reaction of 4-methylbenzenediazonium tetrafluoroborate $(225 \mathrm{mg}$, $1.09 \mathrm{mmol}$ ) and $\mathrm{MeSO}_{2} \mathrm{Na}$ (112 mg, $1.09 \mathrm{mmol}, 1.00$ equiv) in $\mathrm{CH}_{2} \mathrm{Cl}_{2}$ (3.60 mL, $0.30 \mathrm{M}$ ) gave $\mathbf{1 f}\left(75.4 \mathrm{mg}, 3.80 \cdot 10^{-1} \mathrm{mmol}, 35 \%\right.$ ) after recrystallisation from cold $\mathrm{CH}_{2} \mathrm{Cl}_{2} / n$-hexane as a yellow solid; mp 100.2$100.9{ }^{\circ} \mathrm{C}$ (dec.).

IR (KBr, neat): 3043, 2937, 3390, 2073, 1324, 1146, 948, 893, $826 \mathrm{~cm}^{-1}$. ${ }^{1} \mathrm{H} \mathrm{NMR}\left(600 \mathrm{MHz}, \mathrm{CDCl}_{3}\right): \delta=7.85(\mathrm{~d}, J=8.3 \mathrm{~Hz}, 2 \mathrm{H}), 7.37(\mathrm{~d}, J=8.2$ $\mathrm{Hz}, 2 \mathrm{H}), 3.21$ (s, $3 \mathrm{H}), 2.48$ (s, $3 \mathrm{H})$.

${ }^{13} \mathrm{C}$ NMR $\left(151 \mathrm{MHz}, \mathrm{CDCl}_{3}\right): \delta=146.9,130.3,124.7,34.7,21.9$.

MS (EI): $m / z(\%)=65$ (39), 91 (100), $119(27)$.

\section{1-(Methylsulfonyl)-2-(4-Methoxyphenyl)diazene (1g)}

Reaction of 4-methoxybenzenediazonium tetrafluoroborate $(225 \mathrm{mg}$, $1.01 \mathrm{mmol}$ ) and $\mathrm{MeSO}_{2} \mathrm{Na}$ (103 mg, $1.01 \mathrm{mmol}, 1.00$ equiv) in $\mathrm{CH}_{2} \mathrm{Cl}_{2}$ (3.40 mL, $0.30 \mathrm{M}$ ) gave $1 \mathrm{~g}\left(143 \mathrm{mg}, 6.67 \cdot 10^{-1} \mathrm{mmol}, 66 \%\right.$ ) after recrystallisation from cold $\mathrm{CH}_{2} \mathrm{Cl}_{2} / n$-hexane as a yellow solid.

${ }^{1} \mathrm{H} \mathrm{NMR}\left(400 \mathrm{MHz}, \mathrm{CDCl}_{3}\right): \delta=7.95(\mathrm{~d}, J=9.0 \mathrm{~Hz}, 2 \mathrm{H}), 7.04(\mathrm{~d}, J=9.0$ $\mathrm{Hz}, 2 \mathrm{H}), 3.94$ (s, $3 \mathrm{H}), 3.19$ (s, $3 \mathrm{H}$ ).

The NMR data were in accordance with the literature. ${ }^{14 a}$

\section{1-(Methylsulfonyl)-2-(4-Acetylphenyl)diazene (1h)}

Reaction of 4-acetylbenzenediazonium tetrafluoroborate $(1.00 \mathrm{~g}$, $4.27 \mathrm{mmol}$ ) and $\mathrm{MeSO}_{2} \mathrm{Na}$ (437 mg, $4.27 \mathrm{mmol}, 1.00$ equiv) in $\mathrm{CH}_{2} \mathrm{Cl}_{2}$ (14.0 mL, $0.30 \mathrm{M}$ ) gave $\mathbf{1 h}(840 \mathrm{mg}, 3.71 \mathrm{mmol}, 87 \%)$ after recrystallisation from cold $\mathrm{CH}_{2} \mathrm{Cl}_{2} / n$-hexane as a yellow solid.

${ }^{1} \mathrm{H} \mathrm{NMR}\left(600 \mathrm{MHz}, \mathrm{CDCl}_{3}\right): \delta=8.15(\mathrm{~d}, J=8.5 \mathrm{~Hz}, 2 \mathrm{H}), 8.03(\mathrm{~d}, J=8.5$ $\mathrm{Hz}, 2 \mathrm{H}), 3.26$ (s, $3 \mathrm{H}), 2.69$ (s, $3 \mathrm{H})$.

The NMR data were in accordance with the literature. ${ }^{14 a}$ 


\section{1-(Methylsulfonyl)-2-(1,1'biphenyl)diazene (1i)}

Reaction of 1,1'-biphenyldiazonium tetrafluoroborate $(338 \mathrm{mg}$, $1.43 \mathrm{mmol}$ ) and $\mathrm{MeSO}_{2} \mathrm{Na}$ (146 mg, $1.43 \mathrm{mmol}, 1.00$ equiv) in $\mathrm{CH}_{2} \mathrm{Cl}_{2}$ (5.00 mL, $0.30 \mathrm{M})$ gave $\mathbf{1 i}\left(223 \mathrm{mg}, 8.58 \cdot 10^{-1} \mathrm{mmol}, 60 \%\right)$ after recrystallisation from cold $\mathrm{CH}_{2} \mathrm{Cl}_{2} / n$-hexane as an orange solid; mp 115.5$115.9^{\circ} \mathrm{C}$ (dec.).

IR (KBr, neat): 3038, 2932, 2263, 2062, 1482, 1328, 1142, 953, 889 , $848,767,690 \mathrm{~cm}^{-1}$.

${ }^{1} \mathrm{H}$ NMR $\left(600 \mathrm{MHz}, \mathrm{CDCl}_{3}\right): \delta=8.03(\mathrm{~d}, J=8.5 \mathrm{~Hz}, 2 \mathrm{H}), 7.81(\mathrm{~d}, J=8.5$ $\mathrm{Hz}, 2 \mathrm{H}), 7.67$ (d, $J=7.5 \mathrm{~Hz}, 2 \mathrm{H}), 7.51(\mathrm{t}, J=7.6 \mathrm{~Hz}, 2 \mathrm{H}), 7.45(\mathrm{t}, J=$ $7.3 \mathrm{~Hz}, 1 \mathrm{H}), 3.24$ (s, $3 \mathrm{H})$.

${ }^{13} \mathrm{C}$ NMR $\left(151 \mathrm{MHz}, \mathrm{CDCl}_{3}\right): \delta=148.8,148.0,139.0,129.1,128.8$, 128.2, 127.3, 125.2, 34.8 .

MS (EI): $m / z(\%)=181(22), 153(100)$.

\section{1-(Methylsulfonyl)-2-(4-nitrophenyl)diazene (1j)}

Reaction of 4-nitrobenzenediazonium tetrafluoroborate (304 mg, $1.28 \mathrm{mmol}$ ) and $\mathrm{MeSO}_{2} \mathrm{Na}$ (131 mg, $1.28 \mathrm{mmol}, 1.00$ equiv) in $\mathrm{CH}_{2} \mathrm{Cl}_{2}$ (4.30 mL, $0.30 \mathrm{M}$ ) gave $\mathbf{1 j}$ ( $\left.215 \mathrm{mg}, 9.38 \cdot 10^{-1} \mathrm{mmol}, 71 \%\right)$ after recrystallisation from cold $\mathrm{CH}_{2} \mathrm{Cl}_{2} / n$-hexane as a yellow solid.

${ }^{1} \mathrm{H}$ NMR $\left(600 \mathrm{MHz}, \mathrm{CDCl}_{3}\right): \delta=8.45(\mathrm{~d}, J=9.0 \mathrm{~Hz}, 2 \mathrm{H}), 8.12(\mathrm{~d}, J=8.9$ $\mathrm{Hz}, 2 \mathrm{H}), 3.28$ (s, $3 \mathrm{H})$.

The NMR data were in accordance with the literature. ${ }^{14 \mathrm{~b}}$

\section{1-(Methylsulfonyl)-2-[4-pentafluorosulfanyl)phenyl]diazene (1k)} Reaction of 4-(pentafluorosulfanyl)benzenediazonium tetrafluoroborate $(497 \mathrm{mg}, 1.56 \mathrm{mmol})$ and $\mathrm{MeSO}_{2} \mathrm{Na}(160 \mathrm{mg}, 1.56 \mathrm{mmol}, 1.00$ equiv) in $\mathrm{CH}_{2} \mathrm{Cl}_{2}(5.30 \mathrm{~mL}, 0.30 \mathrm{M})$ gave $1 \mathbf{k}\left(281 \mathrm{mg}, 9.06 \cdot 10^{-1} \mathrm{mmol}\right.$, $58 \%)$ after recrystallisation from cold $\mathrm{CH}_{2} \mathrm{Cl}_{2} / n$-hexane as an orange solid; mp $100.4-100.9{ }^{\circ} \mathrm{C}$ (dec.).

IR (KBr, neat): 3019, 2934, 1323, 1142, 963, $825 \mathrm{~cm}^{-1}$.

${ }^{1} \mathrm{H}$ NMR $\left(400 \mathrm{MHz}, \mathrm{CDCl}_{3}\right): \delta=8.07-7.92(\mathrm{~m}, 4 \mathrm{H}), 3.24(\mathrm{~s}, 3 \mathrm{H})$.

${ }^{13} \mathrm{C}$ NMR $\left(101 \mathrm{MHz}, \mathrm{CDCl}_{3}\right): \delta=149.8,127.7,124.4,34.9$.

${ }^{19} \mathrm{~F} \mathrm{NMR}\left(376 \mathrm{MHz}, \mathrm{CDCl}_{3}\right): \delta=83.35-79.34(\mathrm{~m}), 62.52(\mathrm{~d}, J=$ $150.5 \mathrm{~Hz})$.

MS (EI): $m / z(\%)=231$ (35), 203 (100), 95 (69), 75 (61).

\section{1-(Methylsulfonyl)-2-(4-cyanophenyl)diazene (11)}

Reaction of 4-cyanobenzenediazonium tetrafluoroborate $(278 \mathrm{mg}$, $1.28 \mathrm{mmol}$ ) and $\mathrm{MeSO}_{2} \mathrm{Na}\left(131 \mathrm{mg}, 1.28 \mathrm{mmol}, 1.00\right.$ equiv) in $\mathrm{CH}_{2} \mathrm{Cl}_{2}$ $(4.30 \mathrm{~mL}, 0.30 \mathrm{M})$ gave $11(230 \mathrm{mg}, 1.10 \mathrm{mmol}, 86 \%)$ after recrystallisation from cold $\mathrm{CH}_{2} \mathrm{Cl}_{2} / n$-hexane as a yellow solid.

${ }^{1} \mathrm{H} \mathrm{NMR}\left(600 \mathrm{MHz}, \mathrm{CDCl}_{3}\right): \delta=8.05(\mathrm{~d}, J=8.5 \mathrm{~Hz}, 2 \mathrm{H}), 7.90(\mathrm{~d}, J=8.6$ $\mathrm{Hz}, 2 \mathrm{H}), 3.26$ (s, $3 \mathrm{H})$.

The NMR data were in accordance with the literature. ${ }^{14 \mathrm{~b}}$

\section{1-(Methylsulfonyl)-2-(4-ethoxycarbonylphenyl)diazene (1m)}

Reaction of 4-ethoxycarbonylbenzediazonium tetrafluoroborate (431 mg, $1.56 \mathrm{mmol}$ ) and $\mathrm{MeSO}_{2} \mathrm{Na}(160 \mathrm{mg}, 1.56 \mathrm{mmol}, 1.00$ equiv) in $\mathrm{CH}_{2} \mathrm{Cl}_{2}(5.30 \mathrm{~mL}, 0.30 \mathrm{M})$ gave $1 \mathrm{~m}(265 \mathrm{mg}, 1.03 \mathrm{mmol}, 66 \%)$ after column chromatography on silica gel (eluent: 3:1 pentane/EtOAc), followed by recrystallisation from cold $\mathrm{CH}_{2} \mathrm{Cl}_{2} / n$-hexane as a yellow solid; mp 104.6-105.0 ${ }^{\circ} \mathrm{C}$ (dec.).

IR (KBr, neat): 3016, 2988, 2935, 2901, 1717, 1334, 1272, 1148, 1102, $1015,960,895,867,770 \mathrm{~cm}^{-1}$.
${ }^{1} \mathrm{H}$ NMR $\left(600 \mathrm{MHz}, \mathrm{CDCl}_{3}\right): \delta=8.25(\mathrm{~d}, J=8.5 \mathrm{~Hz}, 2 \mathrm{H}), 8.00(\mathrm{~d}, J=8.4$ $\mathrm{Hz}, 2 \mathrm{H}), 4.43$ (q, J = 7.1 Hz, $2 \mathrm{H}), 3.25(\mathrm{~s}, 3 \mathrm{H}), 1.43(\mathrm{t}, J=7.1 \mathrm{~Hz}, 3 \mathrm{H})$.

${ }^{13} \mathrm{C}$ NMR $\left(151 \mathrm{MHz}, \mathrm{CDCl}_{3}\right): \delta=165.1,151.1,130.9,124.2,61.8,34.8$, 14.2.

MS (EI): $m / z(\%)=177(100), 149(11)$.

\section{1-(Methylsulfonyl)-2-(4-methoxycarbonylphenyl)diazene (1n)}

Reaction of 4-methoxycarbonylbenzediazonium tetrafluoroborate (391 mg, $1.56 \mathrm{mmol}$ ) and $\mathrm{MeSO}_{2} \mathrm{Na}(160 \mathrm{mg}, 1.56 \mathrm{mmol}, 1.00$ equiv) in $\mathrm{CH}_{2} \mathrm{Cl}_{2}(5.30 \mathrm{~mL}, 0.30 \mathrm{M})$ gave $1 \mathrm{n}(293 \mathrm{mg}, 1.21 \mathrm{mmol}, 78 \%)$ after column chromatography on silica gel (eluent: 3:1 pentane/EtOAc), followed by recrystallisation from cold $\mathrm{CH}_{2} \mathrm{Cl}_{2} / n$-hexane as a yellow solid; mp $113.0-113.5^{\circ} \mathrm{C}$ (dec.).

IR (KBr, neat): 3016, 2935, 1713, 1435, 1331, 1282, 1155, 1105, 897, $865,773,689 \mathrm{~cm}^{-1}$.

${ }^{1} \mathrm{H}$ NMR $\left(600 \mathrm{MHz}, \mathrm{CDCl}_{3}\right): \delta=8.24(\mathrm{~d}, J=8.5 \mathrm{~Hz}, 2 \mathrm{H}), 8.00(\mathrm{~d}, J=8.5$ $\mathrm{Hz}, 2 \mathrm{H}), 3.98$ (s, $3 \mathrm{H}), 3.25$ (s, $3 \mathrm{H})$.

${ }^{13} \mathrm{C}$ NMR $\left(151 \mathrm{MHz}, \mathrm{CDCl}_{3}\right): \delta=165.6,151.2,135.5,130.9,124.2,52.7$, 34.8.

MS (EI): $m / z(\%)=163$ (90), 135 (100), 103 (37).

\section{1-(Methylsulfonyl)-2-( $\alpha$-naphthalene)diazene (10)}

Reaction of $\alpha$-naphthalenediazonium tetrafluoroborate $(315 \mathrm{mg}$, $1.30 \mathrm{mmol}$ ) and $\mathrm{MeSO}_{2} \mathrm{Na}\left(133 \mathrm{mg}, 1.30 \mathrm{mmol}, 1.00\right.$ equiv) in $\mathrm{CH}_{2} \mathrm{Cl}_{2}$ $(4.30 \mathrm{~mL}, 0.30 \mathrm{M})$ gave $10(286 \mathrm{mg}, 1.22 \mathrm{mmol}, 91 \%)$ after recrystallisation from cold $\mathrm{CH}_{2} \mathrm{Cl}_{2} / n$-hexane as an orange solid; $\mathrm{mp}$ 133.1$133.5^{\circ} \mathrm{C}$ (dec.).

IR (KBr, neat): 3042, 2936, 2074, 1326, 1147, 1087, 1147, 955, 981, $851,806,763 \mathrm{~cm}^{-1}$.

${ }^{1} \mathrm{H} \mathrm{NMR}\left(600 \mathrm{MHz}, \mathrm{CDCl}_{3}\right): \delta=8.68(\mathrm{~d}, J=8.4 \mathrm{~Hz}, 1 \mathrm{H}), 8.18(\mathrm{t}, J=14.9$ $\mathrm{Hz}, 1 \mathrm{H}), 8.03-7.95$ (m, $2 \mathrm{H}), 7.73$ (dd, $J=18.1,10.4 \mathrm{~Hz}, 1 \mathrm{H}), 7.64$ (dt, $J=22.0,7.9 \mathrm{~Hz}, 2 \mathrm{H}), 3.34(\mathrm{~s}, 3 \mathrm{H})$.

${ }^{13} \mathrm{C}$ NMR $\left(151 \mathrm{MHz}, \mathrm{CDCl}_{3}\right): \delta=143.9,136.3,134.3,131.4,128.6$, $128.4,127.3,125.3,122.5,115.1,35.1$.

MS (EI): $m / z(\%)=155$ (100), 143 (76), 127 (28).

\section{1-(Methylsulfonyl)-2-(2-Cyanophenyl)diazene (1p)}

Reaction of 2-cyanobenzenediazonium tetrafluoroborate $(100 \mathrm{mg}$ $4.61 \cdot 10^{-1} \mathrm{mmol}$ ) and $\mathrm{MeSO}_{2} \mathrm{Na}\left(46.7 \mathrm{mg}, 4.61 \cdot 10^{-1} \mathrm{mmol}, 1.00\right.$ equiv) in $\mathrm{CH}_{2} \mathrm{Cl}_{2}(1.50 \mathrm{~mL}, 0.30 \mathrm{M})$ gave $1 \mathbf{p}\left(38.0 \mathrm{mg}, 1.82 \cdot 10^{-1} \mathrm{mmol}, 40 \%\right)$ after recrystallisation from cold $\mathrm{CH}_{2} \mathrm{Cl}_{2} / n$-hexane as a yellow solid; mp $112.1-112.7^{\circ} \mathrm{C}$ (dec.).

IR (KBr, neat): 3455, 3149, 3098, 3036, 3010, 2926, 2852, 2633, 2491, 2339, 2231, 2089, 1989, 1870, 1440, 1647, 1550, 1485, 1421, 1334, $1273,1209,1151,1039,956,887,810,773,746,694 \mathrm{~cm}^{-1}$.

${ }^{1} \mathrm{H}$ NMR $\left(600 \mathrm{MHz}, \mathrm{CDCl}_{3}\right): \delta=7.99-7.88(\mathrm{~m}, 2 \mathrm{H}), 7.85-7.78(\mathrm{~m}, 2 \mathrm{H})$, 3.29 (s, $3 \mathrm{H})$.

${ }^{13} \mathrm{C}$ NMR $\left(151 \mathrm{MHz}, \mathrm{CDCl}_{3}\right): \delta=149.1,135.1,134.1,133.9,117.2$, 116.0, 115.2, 34.6.

MS (EI): $m / z(\%)=102(100), 130(53)$.

\section{Methyl 2-[(Methylsulfonyl)diazenyl]benzoate (1q)}

Reaction of 2-methoxycarbonylbenzenediazonium tetrafluoroborate ( $100 \mathrm{mg}, 0.40 \mathrm{mmol}$ ) and $\mathrm{MeSO}_{2} \mathrm{Na}(40.9 \mathrm{mg}, 0.40 \mathrm{mmol}, 1.00$ equiv) in $\mathrm{CH}_{2} \mathrm{Cl}_{2}(1.50 \mathrm{~mL}, 0.30 \mathrm{M})$ gave $1 \mathbf{q}\left(60.7 \mathrm{mg}, 2.51 \cdot 10^{-1} \mathrm{mmol}, 63 \%\right)$ after recrystallisation from cold $\mathrm{CH}_{2} \mathrm{Cl}_{2} / n$-hexane as a yellow solid; mp 78.8-79. ${ }^{\circ} \mathrm{C}$ (dec.). 
IR ( $\mathrm{KBr}$, neat): 3436, 3101, 3019, 2940, 2848, 2638, 2281, 2163, 2096, 1719, 1586, 1489, 1441, 1319, 1267, 1199, 1141, 1267, 1199, 1141, $1077,958,889,833,765,701 \mathrm{~cm}^{-1}$.

${ }^{1} \mathrm{H}$ NMR $\left(600 \mathrm{MHz}, \mathrm{CDCl}_{3}\right): \delta=7.99(\mathrm{dd}, J=7.2,1.8 \mathrm{~Hz}, 1 \mathrm{H}), 7.75-7.64$ (m, $2 \mathrm{H}), 7.61$ (dd, $J=7.5,1.5 \mathrm{~Hz}, 1 \mathrm{H}), 3.94(\mathrm{~s}, 3 \mathrm{H}), 3.21(\mathrm{~s}, 3 \mathrm{H})$.

${ }^{13} \mathrm{C}$ NMR $\left(151 \mathrm{MHz}, \mathrm{CDCl}_{3}\right): \delta=165.7,147.9,133.5,132.7,130.8$, $130.4,117.5,52.8$.

MS (EI): $m / z(\%)=163(26), 135$ (100).

\section{1-(Methylsulfonyl)-2-(3-nitrophenyl)diazene (1r)}

Reaction of 3-nitrobenzenediazonium tetrafluoroborate $(100 \mathrm{mg}$, $0.42 \mathrm{mmol}$ ) and $\mathrm{MeSO}_{2} \mathrm{Na}$ (42.4 mg, $0.42 \mathrm{mmol}, 1.00$ equiv) in $\mathrm{CH}_{2} \mathrm{Cl}_{2}$ $(1.50 \mathrm{~mL}, 0.30 \mathrm{M})$ gave $1 \mathbf{r}\left(38.6 \mathrm{mg}, 1.82 \cdot 10^{-1} \mathrm{mmol}, 40 \%\right)$ after recrystallisation from cold $\mathrm{CH}_{2} \mathrm{Cl}_{2} / n$-hexane as a yellow solid; mp 120.9$121.4^{\circ} \mathrm{C}$ (dec.).

IR (KBr, neat): 3086, 3019, 2935, 2875, 2652, 2323, 2165, 2113, 1996 , 1969, 1931, 1827, 1755, 1605, 1525, 1330, 1154, 1084, 1002, 957, $918,869,817,790,728,667 \mathrm{~cm}^{-1}$.

${ }^{1} \mathrm{H} \mathrm{NMR}\left(600 \mathrm{MHz}, \mathrm{CDCl}_{3}\right): \delta=8.78(\mathrm{t}, J=2.0 \mathrm{~Hz}, 1 \mathrm{H}), 8.53$ (ddd, $J=$ 8.2, 2.2, $1.0 \mathrm{~Hz}, 1 \mathrm{H}), 8.33-8.24(\mathrm{~m}, 1 \mathrm{H}), 7.83(\mathrm{t}, J=8.1 \mathrm{~Hz}, 1 \mathrm{H}), 3.29$ (s, $3 \mathrm{H})$.

${ }^{13} \mathrm{C}$ NMR $\left(151 \mathrm{MHz}, \mathrm{CDCl}_{3}\right): \delta=149.2,130.8,129.3,119.4,35.1$.

MS (EI): $m / z(\%)=122(100), 151$ (53).

\section{Photoinduced Arylborylation; General Procedure}

A solution of arylazo sulfone $\mathbf{1}(0.10 \mathrm{mmol})$ and the respective diboron 2 (0.20 mmol, 2.00 equiv) in degassed $\mathrm{MeCN}(2.00 \mathrm{~mL}, 0.05 \mathrm{M})$ was poured into a glass vessel, capped, and exposed to blue light (H150 Kessil lamp, $34 \mathrm{~W}, 420 \mathrm{~nm}$ ) at r.t. for $12 \mathrm{~h}$. After the completion of the reaction (detected by TLC), the solvent was removed in vacuo and the crude product was purified via column chromatography on silica gel.

\section{4,4,5,5-Tetramethyl-2-phenyl-1,3,2-dioxaborolane (3a)}

Reaction of 1a (18.4 mg, $0.10 \mathrm{mmol}$ ) and bis(pinacolato)diboron (2a; $50.8 \mathrm{mg}, 0.20 \mathrm{mmol}, 2.00$ equiv) in $\mathrm{MeCN}(2.00 \mathrm{~mL}, 0.05 \mathrm{M})$ gave 3a (10.8 $\mathrm{mg}, 5.29 \cdot 10^{-2} \mathrm{mmol}, 53 \%$ ) after purification by column chromatography on silica gel with a mixture of toluene/EtOAc (30:1) as a colourless oil.

${ }^{1} \mathrm{H} \mathrm{NMR}\left(600 \mathrm{MHz}, \mathrm{CDCl}_{3}\right): \delta=7.81(\mathrm{~d}, J=7.0 \mathrm{~Hz}, 2 \mathrm{H}), 7.46(\mathrm{t}, J=7.4$ $\mathrm{Hz}, 1 \mathrm{H}), 7.37$ (t, J = 7.5 Hz, $2 \mathrm{H}), 1.35(\mathrm{~s}, J=4.6 \mathrm{~Hz}, 12 \mathrm{H})$.

The NMR data were in accordance with the literature. ${ }^{10 \mathrm{~b}}$

\section{2-(4-Fluorophenyl)-4,4,5,5-tetramethyl-1,3,2-dioxaborolane (3b)} Reaction of $\mathbf{1 b}(20.2 \mathrm{mg}, 0.10 \mathrm{mmol})$ and $\mathbf{2 a}(50.8 \mathrm{mg}, 0.20 \mathrm{mmol}$, 2.00 equiv) in $\mathrm{MeCN}(2.00 \mathrm{~mL}, 0.05 \mathrm{M})$ gave $3 \mathbf{b}\left(11.9 \mathrm{mg}, 5.36 \cdot 10^{-2}\right.$ $\mathrm{mmol}, 54 \%)$ after purification by column chromatography on silica gel with a mixture of toluene/EtOAc (30:1) as a colourless solid.

${ }^{1} \mathrm{H} \mathrm{NMR}\left(600 \mathrm{MHz}, \mathrm{CDCl}_{3}\right): \delta=7.66(\mathrm{~d}, J=8.0 \mathrm{~Hz}, 2 \mathrm{H}), 7.50(\mathrm{~d}, J=8.1$ $\mathrm{Hz}, 2 \mathrm{H}), 1.34$ (s, $12 \mathrm{H})$.

The NMR data were in accordance with the literature. ${ }^{17}$

\section{2-(4-Chlorophenyl)-4,4,5,5-tetramethyl-1,3,2-dioxaborolane (3c)}

Reaction of 1c $(21.9 \mathrm{mg}, 0.10 \mathrm{mmol})$ and $\mathbf{2 a}(50.8 \mathrm{mg}, 0.20 \mathrm{mmol}$, 2.00 equiv) in $\mathrm{MeCN}(2.00 \mathrm{~mL}, 0.05 \mathrm{M})$ gave $3 c\left(16.4 \mathrm{mg}, 6.88 \cdot 10^{-2}\right.$ $\mathrm{mmol}, 69 \%$ ) after purification by column chromatography on silica gel with a mixture of toluene/EtOAc (30:1) as a colourless solid.
${ }^{1} \mathrm{H}$ NMR $\left(600 \mathrm{MHz}, \mathrm{CDCl}_{3}\right): \delta=7.73(\mathrm{~d}, J=8.0 \mathrm{~Hz}, 2 \mathrm{H}), 7.34(\mathrm{~d}, J=8.0$ $\mathrm{Hz}, 2 \mathrm{H}), 1.34$ (s, $12 \mathrm{H}$ ).

The NMR data were in accordance with the literature. ${ }^{17}$

2-(4-Bromophenyl)-4,4,5,5-tetramethyl-1,3,2-dioxaborolane (3d) Reaction of 1d $(26.3 \mathrm{mg}, 0.10 \mathrm{mmol})$ and $2 \mathbf{2 a}(50.8 \mathrm{mg}, 0.20 \mathrm{mmol}$, 2.00 equiv) in $\mathrm{MeCN}(2.00 \mathrm{~mL}, 0.05 \mathrm{M})$ gave $3 d$ (16.9 mg, $5.97 \cdot 10^{-2}$ $\mathrm{mmol}, 60 \%)$ after purification by column chromatography on silica gel with a mixture of toluene/EtOAc (30:1) as a white solid.

${ }^{1} \mathrm{H} \mathrm{NMR}\left(600 \mathrm{MHz}, \mathrm{CDCl}_{3}\right): \delta=7.80(\mathrm{t}, J=7.2 \mathrm{~Hz}, 2 \mathrm{H}), 7.05(\mathrm{t}, J=8.8$ $\mathrm{Hz}, 2 \mathrm{H}), 1.35$ (d, $J=8.5 \mathrm{~Hz}, 12 \mathrm{H}$ ).

The NMR data were in accordance with the literature. ${ }^{17}$

2-(4-Iodophenyl)-4,4,5,5-tetramethyl-1,3,2-dioxaborolane (3e)

Reaction of 1e $(26.3 \mathrm{mg}, 0.10 \mathrm{mmol})$ and $2 \mathbf{2}(50.8 \mathrm{mg}, 0.20 \mathrm{mmol}$, 2.00 equiv) in $\mathrm{MeCN}(2.00 \mathrm{~mL}, 0.05 \mathrm{M})$ gave 3 e $\left(16.9 \mathrm{mg}, 5.12 \cdot 10^{-2}\right.$ $\mathrm{mmol}, 51 \%$ ) after purification by column chromatography on silica gel with a mixture of toluene/EtOAc (30:1) as a colourless solid.

${ }^{1} \mathrm{H} \mathrm{NMR}\left(600 \mathrm{MHz}, \mathrm{CDCl}_{3}\right): \delta=7.72(\mathrm{~d}, J=8.0 \mathrm{~Hz}, 2 \mathrm{H}), 7.51(\mathrm{~d}, J=7.9$ $\mathrm{Hz}, 2 \mathrm{H}), 1.33$ (s, $12 \mathrm{H})$.

The NMR data were in accordance with the literature. ${ }^{17}$

2-(4-Methylphenyl)-4,4,5,5-tetramethyl-1,3,2-dioxaborolane (3f) Reaction of 1f $(19.8 \mathrm{mg}, 0.10 \mathrm{mmol})$ and $2 \mathbf{a}(50.8 \mathrm{mg}, 0.20 \mathrm{mmol}$, 2.00 equiv) in $\mathrm{MeCN}(2.00 \mathrm{~mL}, 0.05 \mathrm{M})$ gave $3 f\left(17.0 \mathrm{mg}, 7.79 \cdot 10^{-2}\right.$ $\mathrm{mmol}, 78 \%$ ) after purification by column chromatography on silica gel with a mixture of toluene/EtOAc (30:1) as a colourless oil.

${ }^{1} \mathrm{H}$ NMR $\left(600 \mathrm{MHz}, \mathrm{CDCl}_{3}\right.$ ): $\delta=7.71(\mathrm{~d}, J=7.8 \mathrm{~Hz}, 2 \mathrm{H}$ ), 7.17 (dd, $J=19.6,6.9 \mathrm{~Hz}, 2 \mathrm{H}), 2.37$ (s, $3 \mathrm{H}), 1.34$ (s, $12 \mathrm{H}$ ).

The NMR data were in accordance with the literature. ${ }^{10 \mathrm{~b}}$

\section{2-(4-Methoxyphenyl)-4,4,5,5-tetramethyl-1,3,2-dioxaborolane} (3g)

Reaction of 4-methylphenyl mesylate $(\mathbf{1 g}, 21.4 \mathrm{mg}, 0.10 \mathrm{mmol})$ and bis(pinacolato)diboron (2a, $50.8 \mathrm{mg}, 0.20 \mathrm{mmol}, 2.00$ equiv) in acetonitrile (2.00 mL, $0.05 \mathrm{M})$ gave $3 \mathrm{~g}\left(15.7 \mathrm{mg}, 6.71 \cdot 10^{-2} \mathrm{mmol}, 67 \%\right)$ after purification via column chromatography on silica gel with a mixture of toluene/EtOAc (30:1) as a colourless oil.

${ }^{1} \mathrm{H} \mathrm{NMR}\left(600 \mathrm{MHz}, \mathrm{CDCl}_{3}\right): \delta=7.73(\mathrm{~d}, J=8.4 \mathrm{~Hz}, 2 \mathrm{H}), 6.90(\mathrm{~d}, J=8.5$ $\mathrm{Hz}, 2 \mathrm{H}), 3.83$ (s, $3 \mathrm{H}), 1.33$ (s, $12 \mathrm{H}$ ) ppm.

The NMR data were in accordance with the reported literature. ${ }^{10 a}$

\section{2-(4-Acetylphenyl)-4,4,5,5-tetramethyl-1,3,2-dioxaborolane (3h)} Reaction of $\mathbf{1 h}(22.6 \mathrm{mg}, 0.10 \mathrm{mmol})$ and $\mathbf{2 a}(50.8 \mathrm{mg}, 0.20 \mathrm{mmol}$, 2.00 equiv) in $\mathrm{MeCN}(2.00 \mathrm{~mL}, 0.05 \mathrm{M})$ gave $3 \mathbf{h}\left(12.8 \mathrm{mg}, 5.20 \cdot 10^{-2}\right.$ mmol, $52 \%$ ) after purification by column chromatography on silica gel with a mixture of toluene/EtOAc (30:1) as a white solid.

${ }^{1} \mathrm{H} \mathrm{NMR}\left(400 \mathrm{MHz}, \mathrm{CDCl}_{3}\right): \delta=7.91(\mathrm{~m}, 4 \mathrm{H}), 2.62(\mathrm{~s}, 3 \mathrm{H}), 1.36(\mathrm{~s}, J=$ $7.3 \mathrm{~Hz}, 12 \mathrm{H})$.

The NMR data were in accordance with the literature. ${ }^{10 a}$

\section{2-(1,1'-Biphenyl)-4,4,5,5-tetramethyl-1,3,2-dioxaborolane (3i)}

Reaction of $1 \mathbf{i}(26.0 \mathrm{mg}, 0.10 \mathrm{mmol})$ and $2 \mathbf{a}(50.8 \mathrm{mg}, 0.20 \mathrm{mmol}$, 2.00 equiv) in $\mathrm{MeCN}(2.00 \mathrm{~mL}, 0.05 \mathrm{M})$ gave $3 \mathbf{i}\left(15.9 \mathrm{mg}, 5.68 \cdot 10^{-}\right.$ $2 \mathrm{mmol}, 57 \%$ ) after purification by column chromatography on silica gel with a mixture of toluene/EtOAc (30:1) as a yellow solid. 
${ }^{1} \mathrm{H}$ NMR $\left(600 \mathrm{MHz}, \mathrm{CDCl}_{3}\right): \delta=7.90(\mathrm{~d}, J=8.0 \mathrm{~Hz}, 2 \mathrm{H}), 7.62(\mathrm{dt}, J=$ 11.4, $5.3 \mathrm{~Hz}, 4 \mathrm{H}), 7.45$ (t, J = 7.7 Hz, $2 \mathrm{H}), 7.38-7.34(\mathrm{~m}, 1 \mathrm{H}), 1.37$ (s, $12 \mathrm{H})$.

The NMR data were in accordance with the literature. ${ }^{10 a}$

\section{2-(4-Nitrophenyl)-4,4,5,5-tetramethyl-1,3,2-dioxaborolane (3j)}

Reaction of $\mathbf{1 j}(22.9 \mathrm{mg}, 0.10 \mathrm{mmol})$ and $\mathbf{2 a}(50.8 \mathrm{mg}, 0.20 \mathrm{mmol}$, 2.00 equiv) in $\mathrm{MeCN}(2.00 \mathrm{~mL}, 0.05 \mathrm{M})$ gave $3 \mathbf{j}\left(13.4 \mathrm{mg}, 5.38 \cdot 10^{-2}\right.$ $\mathrm{mmol}, 54 \%$ ) after purification by column chromatography on silica gel with a mixture of toluene/EtOAc (30:1) as a colourless oil.

${ }^{1} \mathrm{H}$ NMR $\left(600 \mathrm{MHz}, \mathrm{CDCl}_{3}\right): \delta=7.88(\mathrm{~d}, J=8.1 \mathrm{~Hz}, 2 \mathrm{H}), 7.73(\mathrm{~d}, J=8.5$ $\mathrm{Hz}, 2 \mathrm{H}), 1.35$ (s, $12 \mathrm{H})$.

The NMR data were in accordance with the literature. ${ }^{18}$

\section{2-[4-Pentafluorosulfanyl)phenyl]-4,4,5,5-tetramethyl-1,3,2-diox- aborolane (3k)}

Reaction of $1 \mathbf{k}(31.0 \mathrm{mg}, 0.10 \mathrm{mmol})$ and $2 \mathbf{a}(50.8 \mathrm{mg}, 0.20 \mathrm{mmol}$, 2.00 equiv) in $\mathrm{MeCN}(2.00 \mathrm{~mL}, 0.05 \mathrm{M})$ gave $3 \mathbf{k}\left(20.8 \mathrm{mg}, 6.30 \cdot 10^{-2}\right.$ $\mathrm{mmol}, 63 \%$ ) after purification by column chromatography on silica gel with a mixture of toluene/EtOAc (30:1) as a yellowish solid.

${ }^{1} \mathrm{H}$ NMR $\left(600 \mathrm{MHz}, \mathrm{CDCl}_{3}\right): \delta=7.88(\mathrm{~d}, J=8.1 \mathrm{~Hz}, 2 \mathrm{H}), 7.73(\mathrm{~d}, J=8.5$ $\mathrm{Hz}, 2 \mathrm{H}), 1.35$ (s, $12 \mathrm{H})$.

The NMR data were in accordance with the literature. ${ }^{19}$

\section{2-(4-Nitrophenyl)-4,4,5,5-tetramethyl-1,3,2-dioxaborolane (31)}

Reaction of 11 (20.9 mg, $0.10 \mathrm{mmol})$ and 2a (50.8 mg, $0.20 \mathrm{mmol}$, 2.00 equiv) in $\mathrm{MeCN}(2.00 \mathrm{~mL}, 0.05 \mathrm{M})$ gave 31 (10.3 $\mathrm{mg}, 4.50 \cdot 10^{-2}$ $\mathrm{mmol}, 45 \%$ ) after purification by column chromatography on silica gel with a mixture of toluene/EtOAc (30:1) as a colourless oil.

${ }^{1} \mathrm{H} \mathrm{NMR}\left(600 \mathrm{MHz}, \mathrm{CDCl}_{3}\right): \delta=7.88(\mathrm{~d}, J=7.9 \mathrm{~Hz}, 2 \mathrm{H}), 7.64(\mathrm{~d}, J=8.0$ $\mathrm{Hz}, 2 \mathrm{H}), 1.35$ (s, $12 \mathrm{H})$.

The NMR data were in accordance with the literature. ${ }^{10 a}$

\section{Ethyl 4-(4,4,5,5-Tetramethyl-1,3,2-dioxaborolan-2-yl)benzoate (3m)}

Reaction of $1 \mathbf{m}$ (25.6 mg, $0.10 \mathrm{mmol}$ ) and $\mathbf{2 a}(50.8 \mathrm{mg}, 0.20 \mathrm{mmol}$, 2.00 equiv) in $\mathrm{MeCN}(2.00 \mathrm{~mL}, 0.05 \mathrm{M})$ gave $3 \mathrm{~m}\left(15.2 \mathrm{mg}, 5.50 \cdot 10^{-}\right.$ ${ }^{2} \mathrm{mmol}, 55 \%$ ) after purification via column chromatography on silica gel with a mixture of toluene/EtOAc (30:1) as a white solid.

${ }^{1} \mathrm{H}$ NMR $\left(600 \mathrm{MHz}, \mathrm{CDCl}_{3}\right): \delta=8.02(\mathrm{~d}, J=8.0 \mathrm{~Hz}, 2 \mathrm{H}), 7.86(\mathrm{~d}, J=7.9$ $\mathrm{Hz}, 2 \mathrm{H}), 4.38$ (q, J = 7.1 Hz, $2 \mathrm{H}), 1.40(\mathrm{t}, J=7.1 \mathrm{~Hz}, 3 \mathrm{H}), 1.36(\mathrm{~s}, 12 \mathrm{H})$. The NMR data were in accordance with the literature. ${ }^{10 a}$

\section{Methyl 4-(4,4,5,5-Tetramethyl-1,3,2-dioxaborolan-2-yl)benzoate (3n)}

Reaction of 1n (24.2 mg, $0.10 \mathrm{mmol})$ and $2 \mathbf{a}(50.8 \mathrm{mg}, 0.20 \mathrm{mmol}$, 2.00 equiv) in $\mathrm{MeCN}(2.00 \mathrm{~mL}, 0.05 \mathrm{M})$ gave $3 \mathrm{n}\left(14.8 \mathrm{mg}, 5.68 \cdot 10^{-2}\right.$ mmol, $57 \%$ ) after purification by column chromatography on silica gel with a mixture of toluene/EtOAc (30:1) as a colourless oil.

${ }^{1} \mathrm{H} \mathrm{NMR}\left(600 \mathrm{MHz}, \mathrm{CDCl}_{3}\right): \delta=8.02(\mathrm{~d}, J=8.1 \mathrm{~Hz}, 2 \mathrm{H}), 7.87(\mathrm{~d}, J=8.1$ $\mathrm{Hz}, 2 \mathrm{H}), 3.92$ (s, $3 \mathrm{H}), 1.35$ (s, $12 \mathrm{H}$ ).

The NMR data were in accordance with the literature. ${ }^{17}$

\section{2-(Naphthalene-1-yl)-4,4,5,5-tetramethyl-1,3,2-dioxaborolane}

(3o)

Reaction of 10 (23.4 mg, $0.10 \mathrm{mmol})$ and $2 \mathrm{a}(50.8 \mathrm{mg}, 0.20 \mathrm{mmol}$, 2.00 equiv) in $\mathrm{MeCN}(2.00 \mathrm{~mL}, 0.05 \mathrm{M})$ gave $30\left(13.3 \mathrm{mg}, 5.23 \cdot 10^{-2}\right.$ $\mathrm{mmol}, 52 \%)$ after purification by column chromatography on silica gel with a mixture of toluene/EtOAc (50:1) as a yellowish solid.

${ }^{1} \mathrm{H} \mathrm{NMR}\left(600 \mathrm{MHz}, \mathrm{CDCl}_{3}\right): \delta=8.76(\mathrm{~d}, J=8.4 \mathrm{~Hz}, 1 \mathrm{H}), 8.76(\mathrm{~d}, J=8.4$ $\mathrm{Hz}, 1 \mathrm{H}), 8.08(\mathrm{~d}, J=6.8 \mathrm{~Hz}, 1 \mathrm{H}), 7.93(\mathrm{~d}, J=8.1 \mathrm{~Hz}, 1 \mathrm{H}), 7.83(\mathrm{~d}, J=8.1$ $\mathrm{Hz}, 1 \mathrm{H}$ ), 7.56-7.50 (m, $1 \mathrm{H}), 7.47$ (t, J = 7.5 Hz, $1 \mathrm{H}), 1.43$ (s, $12 \mathrm{H})$.

The NMR data were in accordance with the literature. ${ }^{9 \mathrm{v}}$

\section{5,5-Dimethyl-2-(p-tolyl)-1,3,2-dioxaborinane (3p)}

Reaction of $\mathbf{1 f}(19.8 \mathrm{mg}, 0.10 \mathrm{mmol})$ and 5,5,5',5'-tetramethyl-2,2'bi(1,3,2-dioxaborinane) (2b; $45.1 \mathrm{mg}, 0.20 \mathrm{mmol}, 2.00$ equiv) in

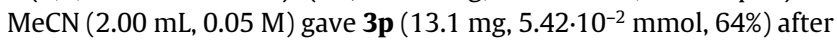
purification by column chromatography on silica gel with a mixture of pentane/EtOAc (30:1) as a white solid.

${ }^{1} \mathrm{H} \mathrm{NMR}\left(600 \mathrm{MHz}, \mathrm{CDCl}_{3}\right): \delta=7.70(\mathrm{~d}, J=7.8 \mathrm{~Hz}, 2 \mathrm{H}), 7.18(\mathrm{~d}, J=7.7$ $\mathrm{Hz}, 2 \mathrm{H}), 3.76$ (s, $4 \mathrm{H}), 2.36$ (s, $3 \mathrm{H}), 1.02$ (s, $6 \mathrm{H}$ ).

The NMR data were in accordance with the literature. ${ }^{\mathrm{k}}$

\section{4,5,5-Trimethyl-2-(p-tolyl)-1,3,2-dioxaborinane (3q)}

Reaction of $\mathbf{1 f}(19.8 \mathrm{mg}, 0.10 \mathrm{mmol})$ and 5,5,5',5',6,6'-hexamethyl2,2'-bi(1,3,2-dioxaborinane) (2c; $50.8 \mathrm{mg}, 0.20 \mathrm{mmol}, 2.00$ equiv) in $\mathrm{MeCN}(2.00 \mathrm{~mL}, 0.05 \mathrm{M})$ gave $3 q\left(12.1 \mathrm{mg}, 5.55 \cdot 10^{-2} \mathrm{mmol}, 56 \%\right)$ after purification by column chromatography on silica gel with a mixture of pentane/EtOAc (30:1) as a colourless oil.

IR (KBr, neat): 2970, 2924, 2330, 2095, 1913, 1610, 1374, 1307, 1165, $815 \mathrm{~cm}^{-1}$.

${ }^{1} \mathrm{H}$ NMR $\left(600 \mathrm{MHz}, \mathrm{CDCl}_{3}\right): \delta=7.70(\mathrm{~d}, J=7.7 \mathrm{~Hz}, 2 \mathrm{H}), 7.15(\mathrm{~d}, J=7.7$ $\mathrm{Hz}, 2 \mathrm{H}), 4.40-4.25$ (m, $1 \mathrm{H}), 2.35$ (s, $3 \mathrm{H}), 1.85$ (dd, $J=13.8,2.9 \mathrm{~Hz}, 1$ H), $1.66-1.52(\mathrm{~m}, 1 \mathrm{H}), 1.35(\mathrm{dd}, J=14.2,6.7 \mathrm{~Hz}, 9 \mathrm{H})$.

${ }^{13} \mathrm{C}$ NMR $\left(151 \mathrm{MHz}, \mathrm{CDCl}_{3}\right): \delta=140.2,133.7,128.2,70.8,64.8,46.0$, $31.4,28.16$ (s), 23.2, $21.6 \mathrm{ppm}$. The carbon directly attached to the boron atom was not detected due to quadrupolar broadening.

${ }^{11} \mathrm{~B} \mathrm{NMR}\left(128 \mathrm{MHz}, \mathrm{CDCl}_{3}\right): \delta=26.8$.

MS (EI): $m / z$ calcd for $\mathrm{C}_{13} \mathrm{H}_{19} \mathrm{BO}_{2}[\mathrm{M}]^{+}:$218.1472; found: 218.1470 .

\section{2-(4-Chlorophenyl)benzo[d][1,3,2]dioxaborole (3r)}

Reaction of $\mathbf{1 c}$ ( $21.9 \mathrm{mg}, 0.10 \mathrm{mmol})$ and bis(catecholato)diboron (2d; $47.6 \mathrm{mg}, 0.20 \mathrm{mmol}, 2.00$ equiv) in $\mathrm{MeCN}(2.00 \mathrm{~mL}, 0.05 \mathrm{M})$ gave the crude product $3 \mathbf{r}$ as a brown solid. Several attempts to purify the product were unsuccessful. For this reason, the yield (35\%) was determined by ${ }^{1} \mathrm{H}$ NMR spectroscopy using dimethyl sulfone as an internal standard.

${ }^{1} \mathrm{H}$ NMR $\left(600 \mathrm{MHz}, \mathrm{CDCl}_{3}\right): \delta=8.01\left(\mathrm{~d}, J=8.1 \mathrm{~Hz}, 1 \mathrm{H}\right.$, product $\left.\mathrm{CH}_{\mathrm{Ar}}\right)$, $7.47\left(\mathrm{~d}, J=8.2 \mathrm{~Hz}, 1 \mathrm{H}\right.$, product $\left.\mathrm{CH}_{\mathrm{Ar}}\right), 7.39(\mathrm{dd}, J=5.8,3.3 \mathrm{~Hz}, 1 \mathrm{H}$, product $\left.\mathrm{CH}_{\mathrm{Ar}}\right), 7.33-7.29\left(\mathrm{~m}, 1 \mathrm{H}\right.$ product $\left.\mathrm{CH}_{\mathrm{Ar}}\right), 3.00(\mathrm{~s}, 6 \mathrm{H}$ internal standard $2 \times \mathrm{CH}_{3}$ ).

Product was confirmed by mass spectra.

MS (EI): $m / z(\%)=230(87)$.

\section{Photoinduced Synthesis of Aryl Thioethers 5; General Procedure}

A solution of arylazo sulfone $\mathbf{1}(0.20 \mathrm{mmol})$ and the respective disulfide 4 ( $0.40 \mathrm{mmol}, 2.00$ equiv) in degassed $\mathrm{MeCN}(2.00 \mathrm{~mL}, 0.1 \mathrm{M})$ was poured into a glass vessel, capped, and exposed to blue light (H150 Kessil lamp, 34 W, $420 \mathrm{~nm}$ ) at r.t. for $12 \mathrm{~h}$. After the completion 
of the reaction (detected by TLC), the solvent was removed in vacuo, and the crude product was purified by column chromatography on silica gel.

\section{Thioanisole (5a)}

Reaction of 1a (36.8 mg, $0.20 \mathrm{mmol}$ ) and dimethyl disulfide (4a; 37.7 $\mathrm{mg}, 0.40 \mathrm{mmol}, 2.00$ equiv) in $\mathrm{MeCN}(2.00 \mathrm{~mL}, 0.1 \mathrm{M})$ gave $\mathbf{5 a}$ (12.8 $\mathrm{mg}, 10.3 \cdot 10^{-2} \mathrm{mmol}, 51 \%$ ) after purification by column chromatography on silica gel with pentane as a colourless oil.

${ }^{1} \mathrm{H}$ NMR $\left(600 \mathrm{MHz}, \mathrm{CDCl}_{3}\right): \delta=7.36-7.22(\mathrm{~m}, 4 \mathrm{H}), 7.14(\mathrm{tt}, J=5.6,1.9$ $\mathrm{Hz}, 1 \mathrm{H}), 2.49$ (s, $3 \mathrm{H})$.

The NMR data were in accordance with the literature. ${ }^{20}$

\section{4-Chlorothioanisole (5b)}

Reaction of 1c (43.7 mg, $0.20 \mathrm{mmol})$ and $\mathbf{4 a}(37.7 \mathrm{mg}, 0.40 \mathrm{mmol}, 2.00$ equiv) in $\mathrm{MeCN}(2.00 \mathrm{~mL}, 0.1 \mathrm{M})$ gave $5 \mathbf{b}\left(21.9 \mathrm{mg}, 1.38 \cdot 10^{-1} \mathrm{mmol}\right.$, $69 \%$ ) after purification by column chromatography on silica gel with pentane as a colourless oil.

${ }^{1} \mathrm{H} \mathrm{NMR}\left(600 \mathrm{MHz}, \mathrm{CDCl}_{3}\right): \delta=7.28-7.21(\mathrm{~m}, 2 \mathrm{H}), 7.21-7.14(\mathrm{~m}, 2 \mathrm{H})$, $2.46(\mathrm{~s}, 3 \mathrm{H})$.

The NMR data were in accordance with the literature. ${ }^{21}$

\section{4-Bromothioanisole (5c)}

Reaction of 1d (52.6 mg, $0.20 \mathrm{mmol})$ and $4 \mathbf{a}(37.7 \mathrm{mg}, 0.40 \mathrm{mmol}$, 2.00 equiv) in $\mathrm{MeCN}(2.00 \mathrm{~mL}, 0.1 \mathrm{M})$ gave $\mathbf{5 c}\left(30.3 \mathrm{mg}, 14.9 \cdot 10^{-2}\right.$ $\mathrm{mmol}, 75 \%$ ) after purification by column chromatography on silica gel with pentane as a colourless solid.

${ }^{1} \mathrm{H} \mathrm{NMR}\left(600 \mathrm{MHz}, \mathrm{CDCl}_{3}\right): \delta=7.47-7.34(\mathrm{~m}, 2 \mathrm{H}), 7.20-7.00(\mathrm{~m}, 2 \mathrm{H})$, $2.47(\mathrm{~s}, 3 \mathrm{H})$.

The NMR data were in accordance with the literature. ${ }^{22}$

\section{4-Iodothioanisole (5d)}

Reaction of 1e (62.0 mg, $0.20 \mathrm{mmol})$ and $4 \mathbf{a}(37.7 \mathrm{mg}, 0.40 \mathrm{mmol}, 2.00$ equiv) in $\mathrm{MeCN}(2.00 \mathrm{~mL}, 0.1 \mathrm{M})$ gave $5 \mathbf{d}\left(29.0 \mathrm{mg}, 11.6 \cdot 10^{-2} \mathrm{mmol}\right.$, $58 \%$ ) after purification by column chromatography on silica gel with pentane as a yellow solid.

${ }^{1} \mathrm{H} \mathrm{NMR}\left(600 \mathrm{MHz}, \mathrm{CDCl}_{3}\right): \delta=7.56-7.51(\mathrm{~m}, 2 \mathrm{H}), 6.98-6.93(\mathrm{~m}, 2 \mathrm{H})$, $2.43(\mathrm{~s}, 3 \mathrm{H})$.

The NMR data were in accordance with the literature. ${ }^{22}$

\section{4-Methylthioanisole (5e)}

Reaction of $\mathbf{1 f}$ (39.7 mg, $0.20 \mathrm{mmol}$ ) and $\mathbf{4 a}(37.7 \mathrm{mg}, 0.40 \mathrm{mmol}, 2.00$ equiv) in $\mathrm{MeCN}(2.00 \mathrm{~mL}, 0.1 \mathrm{M})$ gave 5 e $\left(16.7 \mathrm{mg}, 12.1 \cdot 10^{-2} \mathrm{mmol}\right.$, $60 \%$ ) after purification by column chromatography on silica gel with pentane as a colourless oil.

${ }^{1} \mathrm{H}$ NMR $\left(600 \mathrm{MHz}, \mathrm{CDCl}_{3}\right): \delta=7.24-7.15(\mathrm{~m}, 2 \mathrm{H}), 7.12(\mathrm{~d}, J=8.1 \mathrm{~Hz}, 2$ $\mathrm{H}), 2.47(\mathrm{~s}, J=4.6 \mathrm{~Hz}, 3 \mathrm{H}), 2.33$ (s, $3 \mathrm{H})$.

The NMR data were in accordance with the literature. ${ }^{22}$

\section{4-Methoxythioanisole (5f)}

Reaction of 1 g $(42.9 \mathrm{mg}, 0.20 \mathrm{mmol})$ and $\mathbf{4 a}(37.7 \mathrm{mg}, 0.40 \mathrm{mmol}$, 2.00 equiv) in $\mathrm{MeCN}(2.00 \mathrm{~mL}, 0.1 \mathrm{M})$ gave $\mathbf{5 f}\left(16.2 \mathrm{mg}, 10.5 \cdot 10^{-2}\right.$ $\mathrm{mmol}, 53 \%$ ) after purification by column chromatography on silica gel with pentane as a colourless oil.

${ }^{1} \mathrm{H} \mathrm{NMR}\left(600 \mathrm{MHz}, \mathrm{CDCl}_{3}\right): \delta=7.32-7.21(\mathrm{~m}, 2 \mathrm{H}), 6.92-6.77(\mathrm{~m}, 2 \mathrm{H})$, $3.79(\mathrm{~s}, 3 \mathrm{H}), 2.45(\mathrm{~s}, 3 \mathrm{H})$.

The NMR data were in accordance with the literature. ${ }^{22}$

\section{4-(Methylthio)benzonitrile (5g)}

Reaction of $\mathbf{1 1}(41.8 \mathrm{mg}, 0.20 \mathrm{mmol}$ ) and $\mathbf{4 a}(37.7 \mathrm{mg}, 0.40 \mathrm{mmol}, 2.00$ equiv) in $\mathrm{MeCN}(2.00 \mathrm{~mL}, 0.1 \mathrm{M})$ gave $5 \mathrm{~g}\left(19.3 \mathrm{mg}, 13.1 \cdot 10^{-2} \mathrm{mmol}\right.$, $66 \%$ ) after purification by column chromatography on silica gel with a mixture of pentane/ $/ \mathrm{Et}_{2} \mathrm{O}(50: 1)$ as a yellow solid.

${ }^{1} \mathrm{H}$ NMR $\left(600 \mathrm{MHz}, \mathrm{CDCl}_{3}\right): \delta=7.58-7.51(\mathrm{~m}, 2 \mathrm{H}), 7.32-7.20(\mathrm{~m}, 2 \mathrm{H})$, 2.50 (s, $3 \mathrm{H})$.

The NMR data were in accordance with the literature. ${ }^{23}$

\section{4-Nitrothioanisole (5h)}

Reaction of $\mathbf{1 j}$ ( $45.8 \mathrm{mg}, 0.20 \mathrm{mmol}$ ) and $\mathbf{4 a}$ ( $37.7 \mathrm{mg}, 0.40 \mathrm{mmol}, 2.00$ equiv) in $\mathrm{MeCN}(2.00 \mathrm{~mL}, 0.1 \mathrm{M})$ gave $5 \mathbf{h}\left(21.4 \mathrm{mg}, 12.6 \cdot 10^{-2} \mathrm{mmol}\right.$, $63 \%$ ) after purification by column chromatography on silica gel with a mixture of pentane/ $/ \mathrm{Et}_{2} \mathrm{O}(90: 1)$ as a yellow solid.

${ }^{1} \mathrm{H} \mathrm{NMR}\left(400 \mathrm{MHz}, \mathrm{CDCl}_{3}\right): \delta=8.24-8.04(\mathrm{~m}, 2 \mathrm{H}), 7.34-7.14(\mathrm{~m}, 2 \mathrm{H})$, $2.54(\mathrm{~s}, 3 \mathrm{H})$.

The NMR data were in accordance with the literature. ${ }^{22}$

Pentafluoro[4-(methylthio)phenyl]sulfane (5i)

Reaction of 1k $(62.1 \mathrm{mg}, 0.20 \mathrm{mmol})$ and $\mathbf{4 a}(37.7 \mathrm{mg}, 0.40 \mathrm{mmol}$, 2.00 equiv) in MeCN $(2.00 \mathrm{~mL}, 0.1 \mathrm{M})$ gave $5 \mathbf{i}\left(29.1 \mathrm{mg}, 11.6 \cdot 10^{-2}\right.$ $\mathrm{mmol}, 58 \%$ ) after purification by column chromatography on silica gel with pentane/ $\mathrm{Et}_{2} \mathrm{O}(80: 1)$ as a yellow solid.

${ }^{1} \mathrm{H} \mathrm{NMR}\left(600 \mathrm{MHz}, \mathrm{CDCl}_{3}\right): \delta=7.70-7.58(\mathrm{~m}, 2 \mathrm{H}), 7.29-7.20(\mathrm{~m}, 2 \mathrm{H})$, $2.51(\mathrm{~s}, 3 \mathrm{H})$.

${ }^{19} \mathrm{~F}$ NMR (376 MHz, $\mathrm{CDCl}_{3}$ ): $\delta=87.57-81.95(\mathrm{~m}), 63.35(\mathrm{~d}, J=150.2$ $\mathrm{Hz}$ ).

The NMR data were in accordance with the literature. ${ }^{24}$

\section{4-Acetylthioanisole (5j)}

Reaction of $\mathbf{1 h}(45.3 \mathrm{mg}, 0.20 \mathrm{mmol})$ and $\mathbf{4 a}(37.7 \mathrm{mg}, 0.40 \mathrm{mmol}$, 2.00 equiv) in $\mathrm{MeCN}(2.00 \mathrm{~mL}, 0.1 \mathrm{M})$ gave $5 \mathbf{j}\left(24.6 \mathrm{mg}, 14.8 \cdot 10^{-2}\right.$ $\mathrm{mmol}, 74 \%$ ) after purification by column chromatography on silica gel with pentane as a colourless solid.

${ }^{1} \mathrm{H}$ NMR $\left(600 \mathrm{MHz}, \mathrm{CDCl}_{3}\right): \delta=7.90-7.83(\mathrm{~m}, 2 \mathrm{H}), 7.31-7.20(\mathrm{~m}, 2 \mathrm{H})$, 2.56 (s, $3 \mathrm{H}), 2.51$ (s, $3 \mathrm{H})$.

The NMR data were in accordance with the literature. ${ }^{25}$

\section{Methyl 2-(Methylthio)benzoate (5k)}

Reaction of 1q (48.5 mg, $0.20 \mathrm{mmol})$ and $4 \mathbf{a}(37.7 \mathrm{mg}, 0.40 \mathrm{mmol}$, 2.00 equiv) in $\mathrm{MeCN}(2.00 \mathrm{~mL}, 0.1 \mathrm{M})$ gave $5 \mathbf{k}\left(18.3 \mathrm{mg}, 10.0 \cdot 10^{-2}\right.$ $\mathrm{mmol}, 50 \%$ ) after purification by column chromatography on silica gel with a mixture of pentane/ $/ \mathrm{Et}_{2} \mathrm{O}(90: 1)$ as a colourless solid.

${ }^{1} \mathrm{H} \mathrm{NMR}\left(600 \mathrm{MHz}, \mathrm{CDCl}_{3}\right): \delta=7.99(\mathrm{dd}, J=7.8,1.5 \mathrm{~Hz}, 1 \mathrm{H}), 7.50-7.44$ (m, $1 \mathrm{H}), 7.29-7.27(\mathrm{~m}, 1 \mathrm{H}), 7.17-7.13(\mathrm{~m}, 1 \mathrm{H}), 3.93-3.90(\mathrm{~m}, 3 \mathrm{H})$, $2.46(\mathrm{~s}, 3 \mathrm{H})$.

The NMR data were in accordance with the literature. ${ }^{21}$

\section{2-(Methylthio)benzonitrile (5l)}

Reaction of 1p (41.8 mg, $0.20 \mathrm{mmol})$ and $4 a(37.7 \mathrm{mg}, 0.40 \mathrm{mmol}$, 2.00 equiv) in $\mathrm{MeCN}(2.00 \mathrm{~mL}, 0.1 \mathrm{M})$ gave 51 (14.5 mg, $14.5 \cdot 10^{-2}$ mmol, $72 \%$ ) after purification by column chromatography on silica gel with a mixture of pentane/ $\mathrm{Et}_{2} \mathrm{O}(90: 1)$ as a colourless solid.

${ }^{1} \mathrm{H}$ NMR $\left(600 \mathrm{MHz}, \mathrm{CDCl}_{3}\right): \delta=7.59(\mathrm{dd}, J=7.7,1.3 \mathrm{~Hz}, 1 \mathrm{H}), 7.53-7.50$ $(\mathrm{m}, 1 \mathrm{H}), 7.32(\mathrm{~d}, J=8.1 \mathrm{~Hz}, 1 \mathrm{H}), 7.23-7.20(\mathrm{~m}, 1 \mathrm{H}), 2.56(\mathrm{~s}, 3 \mathrm{H})$.

The NMR data were in accordance with the literature. ${ }^{22}$ 


\section{3-Nitrothioanisole (5m)}

Reaction of $1 \mathrm{~s}$ ( $33.8 \mathrm{mg}, 0.20 \mathrm{mmol}$ ) and $4 \mathbf{a}(37.7 \mathrm{mg}, 0.40 \mathrm{mmol}, 2.00$ equiv) in $\mathrm{MeCN}(2.00 \mathrm{~mL}, 0.1 \mathrm{M})$ gave $5 \mathrm{~m}\left(18.2 \mathrm{mg}, 10.8 \cdot 10^{-2} \mathrm{mmol}\right.$, $54 \%$ ) after purification by column chromatography on silica gel with a mixture of pentane/ $/ \mathrm{Et}_{2} \mathrm{O}$ as a yellow solid.

${ }^{1} \mathrm{H}$ NMR $\left(600 \mathrm{MHz}, \mathrm{CDCl}_{3}\right): \delta=8.05(\mathrm{t}, J=1.9 \mathrm{~Hz}, 1 \mathrm{H}), 7.96$ (ddd, $J=$ 5.8, 1.9, 0.7 Hz, $1 \mathrm{H}), 7.55-7.51(\mathrm{~m}, 1 \mathrm{H}), 7.43(\mathrm{t}, J=8.0 \mathrm{~Hz}, 1 \mathrm{H}), 2.56$ (s, $3 \mathrm{H})$.

The NMR data were in accordance with the literature. ${ }^{21}$

\section{Ethyl Phenyl Sulfide (5n)}

Reaction of $1 \mathbf{a}$ (36.8 mg, $0.20 \mathrm{mmol}$ ) and diethyl disulfide (4b; $49 \mathrm{mg}$, $0.40 \mathrm{mmol}, 2.00$ equiv) in $\mathrm{MeCN}(2.00 \mathrm{~mL}, 0.1 \mathrm{M})$ gave $5 \mathbf{n}(13.9 \mathrm{mg}$, $10.1 \cdot 10^{-2} \mathrm{mmol}, 50 \%$ ) after purification by column chromatography on silica gel with pentane as a colourless oil.

${ }^{1} \mathrm{H} \mathrm{NMR}\left(400 \mathrm{MHz}, \mathrm{CDCl}_{3}\right): \delta=7.40-7.25(\mathrm{~m}, 4 \mathrm{H}), 7.22-7.11(\mathrm{~m}, 1 \mathrm{H})$, $2.95(\mathrm{q}, J=7.4 \mathrm{~Hz}, 2 \mathrm{H}), 1.32$ (dd, $J=9.2,5.5 \mathrm{~Hz}, 3 \mathrm{H})$.

The NMR data were in accordance with the literature. ${ }^{25}$

\section{Diphenyl Sulfide (5o)}

Reaction of 1a (36.8 mg, $0.20 \mathrm{mmol}$ ) and diphenyl disulfide (4c; 87.3 $\mathrm{mg}, 0.40 \mathrm{mmol}, 2.00$ equiv) in $\mathrm{MeCN}(2.00 \mathrm{~mL}, 0.1 \mathrm{M})$ gave 50 (17.2 $\mathrm{mg}, 9.23 \cdot 10^{-2} \mathrm{mmol}, 46 \%$ ) after purification by column chromatography on silica gel with pentane as a colourless oil.

${ }^{1} \mathrm{H}$ NMR (400 MHz, $\left.\mathrm{CDCl}_{3}\right): \delta=7.70-6.70(\mathrm{~m}, 10 \mathrm{H})$.

The NMR data were in accordance with the literature. ${ }^{19}$

\section{Supporting Information}

Supporting information for this article is available online at https://doi.org/10.1055/s-0037-1611648.

\section{References}

(1) (a) Miyaura, N.; Suzuki, A. Chem. Rev. 1995, 95, 2457. (b) Jana, R.; Pathak, T. P.; Sigman, M. S. Chem. Rev. 2011, 111, 1417. (c) Yamaguchi, J.; Yamaguchi, A. D.; Itami, K. Angew. Chem. Int. Ed. 2012, 51, 8960. (d) Chauhan, P.; Mahajan, S.; Enders, D. Chem. Rev. 2014, 114, 8807. (e) Xu, L.; Zhang, S.; Li, P. Chem. Soc. Rev. 2015, 44, 8848. (f) Fang, Y.; Luo, Z.; Xu, X. RSC Adv. 2016, 6 , 59661. (g) Shen, C.; Zhang, P.; Sun, Q.; Bai, S.; Hor, T. S. A.; Lui, X. Chem. Soc. Rev. 2015, 44, 291.

(2) (a) Ishihara, S. O. K.; Yamamoto, H. J. Org. Chem. 1996, 61, 4196. (b) Antilla, J. C.; Wulff, W. D. J. Am. Chem. Soc. 1999, 121, 5099. (c) Yamamoto, H.; Ishihara, K.; Maki, T. Synlett 2004, 8, 1355. (d) Tang, P. Org. Synth. 2005, 81, 262. (e) Stepanenko, V.; OrtizMarciales, M.; Correa, W.; Jesus, M. D.; Espinosa, S.; Ortiz, L. Tetrahedron: Asymmetry 2006, 17, 112. (f) Newman, C. A.; Antilla, J. C.; Chen, P.; Predeus, A. V.; Fielding, L.; Wulff, W. D. J. Am. Chem. Soc. 2007, 121, 7216. (g) Mellah, M.; Voiturriez, A.; Schulz, E. Chem. Rev. 2007, 107, 5133.

(3) Brooks, W. L.; Sumerlin, B. S. Chem. Rev. 2016, 116, 1375.

(4) (a) Alley, M. R.; Baker, S. J.; Beutner, K. R.; Plattner, J. Expert Opin. Investig. Drugs 2007, 16, 157. (b) Hui, X.; Baker, S. J.; Wester, R. C.; Barbadillo, S.; Cashmore, A. K.; Sanders, V.; Hold, K. M.; Akama, T.; Zhang, Y. K.; Plattner, J. J.; Maibach, H. I. J. Pharm. Sci. 2007, 96, 2622. (c) Vernon, M. W.; Heel, R. C.; Brogden, R. N. Drugs 1991, 42, 997. (d) Conlon, D. A.; Drahus-
Paone, A.; Ho, G. J.; Pipik, B.; Helmy, R.; McNamara, J. M.; Shi, Y. J.; Williams, J. M.; Macdonald, D.; Deschenes, D.; Gallant, M.; Mastracchio, A.; Roy, B.; Scheigetz, J. Org. Process Res. Dev. 2006, 10, 36. (e) Jarvest, R. L.; Berge, J. M.; Brown, M. J.; Brown, P.; Elder, J. S.; Forrest, A. K.; Houge-Frydrych, C. S. V.; O'Hanlon, P. J.; McNair, D. J.; Rittenhouse, S.; Sheppard, R. J. Bioorg. Med. Chem. Lett. 2003, 13, 665.

(5) (a) Waldrop, F. N.; Robertson, R. H.; Vourlekis, A. Compr. Psychiatry 1961, 2, 96. (b) Brogden, R. N.; Heel, R. C.; Speight, T. M.; Avery, G. S. Drugs 1978, 16, 97. (c) Schumacher, D. P.; Clark, J. E.; Murphy, B. L.; Fischer, P. A. J. Org. Chem. 1990, 55, 5291. (d) Pallett, K. E.; Little, J. P.; Veerasekaran, P.; Viviani, F. Pestic. Sci. 1997, 50, 83. (e) Lu, W. Y.; Chen, P.; Lin, G. Q. Tetrahedron 2008, 64, 7822. (f) Van Soolingen, D.; Hernandez-Pando, R.; Orozco, H.; Aguilar, D.; Magis-Escurra, C.; Amaral, L.; van Ingen, J.; Boeree, M. J. PLoS One 2010, 5, e12640. (g) Dunbar, L.; Scharf, D. H.; Litomska, A.; Hertweck, C. Chem. Rev. 2017, 117, 5521.

(6) (a) Kane, R. C.; Bross, P. F.; Farrell, A. T.; Padzur, R. Oncologist 2003, 8, 508. (b) Feng, M.; Tang, B.; Liang, S. H.; Jiang, X. Curr. Top. Med. Chem. 2016, 16, 1200.

(7) (a) Trippler, P. C.; McGuigan, C. Med. Chem. Commun. 2010, 1, 183. (b) Ban, H. S.; Nakamura, H. Chem. Rec. 2015, 15, 616.

(8) (a) Brown, H. C.; Cole, T. E. Organometallics 1983, 2, 1316. (b) Brown, H. C.; Srebnik, M.; Cole, T. E. Organometallics 1986, 5, 2300. (c) Baron, O.; Knochel, P. Angew. Chem. Int. Ed. 2005, 44, 3133.

(9) (a) Ishiyama, T.; Murata, M.; Miyaura, N. J. Org. Chem. 1995, 60, 7508. (b) Chen, H.; Schlecht, S.; Semple, T. C.; Hartwig, J. F. Science 2000, 287, 1995. (c) Shimada, S.; Batsanov, A. S.; Howard, J. A. K.; Marder, T. B. Angew. Chem. Int. Ed. 2001, 40, 2168. (d) Ishiyama, T.; Takagi, J.; Ishida, K.; Miyaura, N.; Anastasi, N. R.; Hartwig, J. F. J. Am. Chem. Soc. 2002, 124, 390. (e) Ishiyama, T.; Miyaura, N. J. Organomet. Chem. 2003, 680, 3. (f) Adams, C. J.; Baber, R. A.; Batsanov, A. S.; Bramham, G.; Charmant, J. P. H.; Haddow, M. F.; Howard, J. A. K.; Lam, W. H.; Lin, Z.; Marder, T. B.; Norman, N. C.; Orpen, A. G. Dalton Trans. 2006, 11, 1370. (g) Zhu, W.; Ma, D. Org. Lett. 2006, 8, 261. (h) Billingsley, K. L.; Barder, T. E.; Buchwald, S. L. Angew. Chem. Int. Ed. 2007, 46, 5359. (i) Rosen, B. M.; Huang, C.; Percec, V. Org. Lett. 2008, 10, 2597. (j) Kleeberg, C.; Dang, L.; Lin, Z.; Marder, T. B. Angew. Chem. Int. Ed. 2009, 48, 5350. (k) Wilson, D. A.; Wilson, C. J.; Moldoveanu, C.; Resmerita, A. M.; Corcoran, P.; Hoang, L. M.; Rosen, B. M.; Percec, V.J. Am. Chem. Soc. 2010, 132, 1800. (l) Mkhalid, I. A. I.; Barnard, J. H.; Marder, T. B.; Murphy, J. M.; Hartwig, J. F. Chem. Rev. 2010, 110, 890. (m) Moldoveanu, C.; Wilson, D. A.; Wilson, C. J.; Leowanawat, P.; Resmerita, A.-M.; Liu, C.; Rosen, B. M.; Percec, V. J. Org. Chem. 2010, 75, 5438. (n) Chow, W. K.; So, C. M.; Lau, C. P.; Kwong, F. Y. Chem. Eur. J. 2011, 17, 6913. (o) Kawamorita, S.; Ohmiya, H.; Iwai, T.; Sawamura, M. Angew. Chem. Int. Ed. 2011, 50, 8363. (p) Yamamoto, T.; Morita, T.; Takagi, J.; Yamakawa, T. Org. Lett. 2011, 13, 5766. (q) Huang, K.; Yu, D.-G.; Zheng, S.-F.; Wu, Z.-H.; Shi, Z.-J. Chem. Eur. J. 2011, 17, 786. (r) Molander, G. A.; Trice, S. L. J.; Kennedy, S. M. J. Org. Chem. 2012, 77, 8678. (s) Chow, W. K.; Yuen, O. Y.; Choy, P. Y.; So, C. M.; Lau, C. P.; Wong, W. T.; Kwong, F. Y. RSC Adv. 2013, 3, 12518. (t) Molander, G. A.; Cavalcanti, L. N.; Garcia-Garcia, C. J. Org. Chem. 2013, 78, 6427. (u) Marciasini, L. D.; Richy, N.; Vaultier, M.; Pucheault, M. Adv. Synth. Catal. 2013, 355, 1083. (v) Bose, S. K.; Marder, T. B. Org. Lett. 2014, 16, 4562. (w) Zarate, C.; Manzano, R.; Martin, R. J. Am. Chem. Soc. 2015, 137, 6754. (x) Frank, R.; Howell, J.; Campos, J.; Tirfoin, R.; Phillips, N.; Zahn, S.; Mingos, D. M. P.; 
Aldridge, S. Angew. Chem. Int. Ed. 2015, 54, 9586. (y) Bhanuchandra, M.; Baralle, A.; Otsuka, S.; Nogi, K.; Yorimitsu, H. Org. Lett. 2016, 18, 2966.

(10) (a) Chen, K.; Zhang, S.; He, P.; Li, P. Chem. Sci. 2016, 7, 3676. (b) Mfuh, A. M.; Doyle, J. D.; Chherti, B.; Arman, H.-D.; Larinov, O. V. J. Am. Chem. Soc. 2016, 138, 2985. (c) Mfuh, A. M.; Schneider, B. D.; Cruces, W.; Larinov, O. V. Nat. Protoc. 2017, 12, 604. (d) Liu, B.; Lim, C.-H.; Miyake, G. M. J. Am. Chem. Soc. 2017, 139, 13616.

(11) Liu, W.; Yang, X.; Gao, Y.; Li, C. J. J. Am Chem. Soc. 2017, 139, 8621.

(12) Candish, L.; Teders, M.; Glorius, F. J. Am. Chem. Soc. 2017, 139, 7440.

(13) For selected examples, see: (a) Deronzier, A.; Cano-Yelo, H. J. Chem. Soc., Perkin Trans. 2 1984, 1093. (b) Hari, D. P.; Schroll, P.; König, B. J. Am. Chem. Soc. 2012, 134, 2958. (c) Hari, D. P.; Hering, T.; König, B. Org. Lett. 2012, 14, 5334. (d) Xiao, T.; Dong, X.; Tang, Y.; Zhou, L. Adv. Synth. Catal. 2012, 354, 3195. (e) Schroll, P.; Hari, D. P.; König, B. ChemistryOpen 2012, 1, 130. (f) Jiang, H.; Huang, C.; Guo, J.; Zeng, C.; Zhang, Y.; Yu, S. Chem. Eur. J. 2012, 18, 15158. (g) Hering, T.; Hari, D. P.; König, B. J. Org. Chem. 2012, 77, 10347. (h) Yu, J.; Zhang, L.; Yan, G. Adv. Synth. Catal. 2012, 354, 2625. (i) Hari, D. P.; Hering, T.; König, B. Angew. Chem. Int. Ed. 2014, 53, 725. (j) Jia, Z. H.; Zhao, C. J.; Zhang, Y. Y.; Xue, D.; Wang, C.; Xiao, J. Chem. Eur. J. 2014, 20, 2960. (k) Zheng, J.; Chu, J.; Zhang, X.; Lei, X. J. Org. Chem. 2014, 79, 10682. (l) Mary, P.; Kundu, D.; Ranu, B. C. Eur. J. Org. Chem. 2015, 1727. (m) Hopkinson, M. N.; Tlahuxt, A.; Glorius, F. Acc. Chem. Res. 2016, 49, 2261. (n) Majek, M.; von Wangelin, J. A. Chem. Commun. 2013, 49, 5507. (o) Li, Y.; Xie, W.; Jiang, X. Chem. Eur. J.
2015, 21, 16059. (p) Ghosh, I.; Marzo, L.; Das, A.; Shaikh, R.; König, B. Acc. Chem. Res. 2016, 49, 1566. (q) Hong, B.; Lee, J.; Lee, A. Tetrahedron Lett. 2017, 58, 2809. (r) Hernández, J. G. Beilstein J. Org. Chem. 2017, 13, 1463.

(14) (a) Crespi, S.; Protti, S.; Fagnoni, M. J. Org. Chem. 2016, 81, 9612. (b) Dossena, A. D.; Sampaolesi, S.; Palmieri, A.; Protti, S.; Fagnoni, M. J. Org. Chem. 2017, 82, 10687. (c) Sauer, C.; Liu, Y.; De Nisi, A.; Protti, S.; Fagnoni, M.; Bandini, M. ChemCatChem 2017, 9, 4456. (d) da Silva Júnior, P. E.; Amin, H. I. M.; Nauth, A. M.; da Silva Emery, F.; Protti, S.; Opatz, T. ChemPhotoChem 2018, 2,878 .

(15) During the preparation of this manuscript a similar protocol for the borylation reaction appeared: Xu, Y.; Yang, X.; Fang, H. J. Org. Chem. 2018, 83, 12831.

(16) Li, Y.; Xie, W.; Jiang, X. Chem. Eur. J. 2015, 21, 16059.

(17) Zhang, L.; Jiao, L. J. Am. Chem. Soc. 2017, 139, 607.

(18) Chow, W. K.; Yuen, O. Y.; So, C. M.; Wong, W. T.; Kwong, F. Y. J. Org. Chem. 2012, 77, 3543.

(19) Benquing, C.; Shichong, J.; Etsuko, T.; Norimichi, S.; Norio, S. Chem. Commun. 2017, 53, 12738.

(20) Harrison, D. J.; Tam, N. C.; Vogels, C. M.; Langler, R. F.; Westcott, S. A. Tetrahedron Lett. 2004, 45, 8493.

(21) Majekt, M.; von Wangelin, A. J. Chem. Commun. 2013, 49, 5507.

(22) Ebenezer, J. M.; Jakob, M. Tetrahedron Lett. 2014, 55, 5323.

(23) Beier, P.; Pestyrikova, T.; Vida, N.; Iakobson, G. Org. Lett. 2011, $13,1466$.

(24) Liu, G.-Z.; Xu, H.-W.; Wang, P.; Lin, Z.-T.; Duan, Y.-C.; Zheng, J.-X.; Liu, H.-M. Eur. J. Med. Chem. 2013, 65, 323.

(25) Gul, K.; Narayanaperumal, S.; Dornelles, L.; Rodriges, O. E. D.; Braga, A. L. Tetrahedron Lett. 2011, 52, 3592. 